\title{
BARGAINING AND WEALTH ACCUMULATION
}

\section{Byeongiu Jeong}

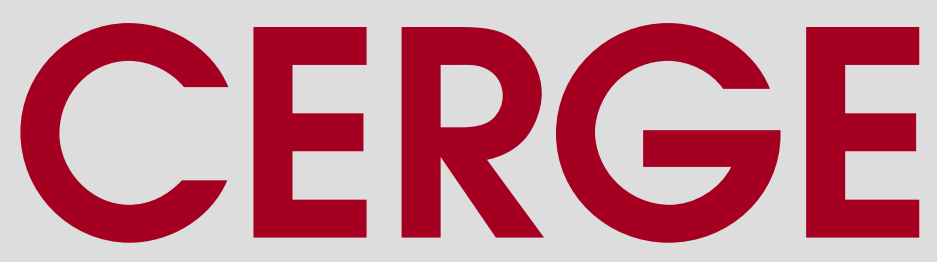




\title{
Working Paper Series $\quad 479$ (ISSN 1211-3298)
}

\section{Bargaining and Wealth Accumulation}

\author{
Byeongju Jeong
}

CERGE-EI

Prague, January 2013 
ISBN 978-80-7343-283-6 (Univerzita Karlova. Centrum pro ekonomický výzkum a doktorské studium)

ISBN 978-80-7344-275-0 (Národohospodářský ústav AV ČR, v.v.i.) 


\title{
Bargaining and Wealth Accumulation
}

\section{Byeongju Jeong*}

\author{
CERGE-EI ${ }^{\dagger}$
}

January 2013

\begin{abstract}
I present a model in which randomly matched pairs of people bargain over the division of output in each period. Output can be consumed or stored for later consumption. People are identical except possibly in wealth (i.e., the stored output). The one-period utility is linear except for the starvation disutility (i.e., the additional drop in utility under no consumption). The starvation disutility weakens the bargaining position of a poor person and strengthens that of a rich person in an otherwise symmetric bargaining, providing the incentive to accumulate wealth. Policies that deincentivize wealth accumulation (e.g., wealth tax, progressive income tax) can make both the rich and the poor become better off.
\end{abstract}

\begin{abstract}
Abstrakt
Prezentuji model, v němž náhodně vytvořené páry opakovaně smlouvají v každém období o rozdělení produktu. Produkt může být spotřebován nebo uchován pro pozdější spotřebu. Lidé jsou identičtí s možnou výjimkou v podobě jejich bohatství (tj. množství produktu uchovaného z předchozích období). Jednoperiodová užitková funkce je lineární ve spotřebě s výjimkou ztráty užitku z hladovění (tj. dodatečný pokles užitku v př́ípadě nulové spotřeby). Tato ztráta užitku oslabuje vyjednávající pozici chudých a posiluje pozici bohatých. To narušuje jinak symetrický charakter smlouvání a poskytuje motivaci k akumulaci bohatství. Politiky, které snižují tuto motivaci (např. daň z bohatství, progresivní př́íjmová daň), můžou dosáhnout zlepšení užitku bohatých i chudých.
\end{abstract}

JEL classification: C78; D31; E21; H21

Keywords: bargain; wealth accumulation, starvation disutility, wealth tax, income tax

\footnotetext{
* I benefited from many comments on the previous drafts. In particuar, I thank Avner Shaked for many long discussions without implicating him on the contents. I also thank Jakub Steiner and Peter Katuscak who read the drafts for the working paper.

${ }^{\dagger}$ CERGE-EI is a joint workplace of the Center for Economic Research and Graduate Education, Charles University, and the Economics Institute of Academy of Sciences of the Czech Republic.

Address: CERGE-EI, P.O. Box 882, Politických vězňů 7, Prague 1, 111 21, Czech Republic
} 


\section{Introduction}

In this paper, I consider bargaining advantages as a possible motive of accumulating wealth. Wealth provides the wealth-holder a degree of security in case of no current income, thereby strengthening the bargaining position of the wealth-holder in dividing the income from a joint production. I model the sense of security in a particular way: The utility is linear in consumption, except that there is a minimum consumption below which there is an added drop in utility, which I call the starvation disutility. I consider the starvation disutility as a simple but realistic metaphor that underlines the prospect of a person with no wealth or current income. It also allows me to focus on the bargaining motive of wealth accumulation aside from precautionary saving (i.e., self-insurance under uncertain marginal utility of consumption), since in equilibrium everyone is guaranteed the minimum consumption and the marginal utility of consumption is constant. I also assume that wealth is a stored consumption good and not a productive asset in order to abstract from the investmentreturn motive of wealth accumulation. The welfare implication of the bargaining motive of wealth accumulation contrasts with that of the other motives in that a person's bargaining advantage is another person's disadvantage. This provides a rationale for policies such as a wealth tax and a progressive income tax.

That a person with no wealth has a weaker bargaining position in relation to a person with some wealth can be understood in terms of risk preference: A more risk-averse person has more to lose from the failure of reaching an agreement, so has a weaker bargaining position. Kihlstrom, Roth, and Schmeidler (1981) shows that the more risk-averse party receives a lower share of surplus in Nash bargaining: If person A's utility over the share of the surplus is an increasing and concave function of person B's utility, person A receives a lower share of surplus than person B in an otherwise symmetric Nash bargaining. In the model of this paper, the one-period utility function over consumption is assumed to 
be identical across the population, but the presence of the starvation utility leads to an endogenously greater risk-averse preference over income for a person with less wealth.

In Krusell, Mukoyama, and Sahin (2010) and Bils, Chang, and Kim (2011), each worker has the one-period utility function over consumption that exhibits the constant relative risk aversion (i.e., $u(c)=\left(c^{1-\gamma}-1\right) /(1-\gamma)$ with $\left.\gamma<1\right)$, bargains over the wage with the risk-neutral firm in each period, and can accumulate wealth. A worker with more wealth becomes "locally" less risk-averse and thus improves his bargaining position and obtains a higher wage. Thus there is a bargaining motive of accumulating wealth, which is not (explicitly) recognized in the articles. The authors' objective is to study the aggregate properties of the models under the productivity and unemployment shocks, which leads to the investment-return and the precautionary motives as well as the bargaining motive of accumulating wealth. The bargaining motive of wealth accumulation appears to play a minor role in their quantitative exercises. As mentioned, in the model of this paper, there are no investment-return or precautionary motives of wealth accumulation. Also, production is modeled in terms of a decentralized random matching among identical people except possibly in wealth. The intent is to portray the bargaining motive of wealth accumulation and its welfare property, abstracting from the other motives of wealth accumulation or the institutional arrangements that may cloud its logic.

This paper can be viewed as a variation in the literature that studies the concern for a person's relative standing in the society. A common approach is to model relative consumption. The concern for relative consumption may lead to socially excessive consumption and, consequently, to the underaccumulation of wealth as argued by Frank (1985). However, there could be an overaccumulation of wealth in order to finance excessive consumption in the future, as in Corneo and Jeanne (1998). In Konrad (1992), people care about their relative wealth, which implies a negative externality of a person's wealth accumulation on the others. Thus wealth accumulation is socially excessive. In Cole, Mailath, and Postlewaite (1992), relative wealth determines the marriage prospects, 
leading to an overaccumulation of wealth absent social restrictions on the marriage patterns. In this paper, relative wealth matters because the person with a greater wealth has the advantage in bargaining with a person with less wealth.

\section{The Model Economy}

There are a large number of people. The size of the population is normalized to one. Each person is randomly matched with another person every day. Each pair of matched people can produce three apples. The two partners of a match bargain over the division of the produced apples. An apple is indivisible. Each person's one-period expected utility is $E[U(c)]$ where $U(c)=c \cdot I(c \geq 1)-\bar{u} \cdot I(c=0)$. Function $I$ is an indicator function that takes on the value of one if the stated condition holds, and zero otherwise. The parameter $\bar{u}$ has a positive value and means the starvation disutility. Each person has the access to a storage technology with the storage capacity of one apple. Each stored apple depreciates fully with probability $\delta$ overnight. The one-period budget constraint is $c+s=w+y$ where $y$ is the number of apples earned today; $w$ is equal to one if an apple was stored yesterday and survived overnight, and to zero otherwise; and $s$ is equal to one if an apple is stored today, and to zero otherwise.

The expected discounted utility of a person with wealth $w$ at the beginning of a period can be written as:

$$
V(w)=\sum_{w^{\prime}}\left\{\lambda\left(w^{\prime}\right) \sum_{y} \pi\left(y ; w, w^{\prime}\right) \max _{s \in \Upsilon(w+y)}\{u(w+y-s)+\tilde{V}(s)\}\right\}
$$

where $w, w^{\prime} \in\{0,1\} ; \lambda\left(w^{\prime}\right)$ is the share of population with $w^{\prime}$ apples; $\pi\left(y ; w, w^{\prime}\right)$ is the probability of taking $y$ apples from production, given the person's wealth $w$ and the partner's wealth $w^{\prime} ; \Upsilon(w+y) \equiv\{0, \min \{1, w+y\}\}$; and $\tilde{V}(s)$ is the utility of the person with wealth $s$ at the end of the period:

$$
\tilde{V}(0)=\beta V(0)
$$




$$
\tilde{V}(1)=\beta \delta \cdot V(0)+\beta(1-\delta) \cdot V(1)
$$

The parameter $\beta$ is the discount rate.

I assume the bargaining outcome to maximize the Nash product: for each $\left(w, w^{\prime}\right)$, $\left\{\pi\left(y ; w, w^{\prime}\right)\right\}_{y}$ solves

$$
\max \left\{\left(\sum_{y} \pi\left(y ; w, w^{\prime}\right) \cdot W(w+y)-W(w)\right)\left(\sum_{y} \pi\left(y ; w, w^{\prime}\right) \cdot W\left(w^{\prime}+3-y\right)-W\left(w^{\prime}\right)\right)\right\}
$$

subject to $\sum_{y} \pi\left(y ; w, w^{\prime}\right)=1$, where $W(w+y) \equiv \max _{s \in \Upsilon(w+y)}\{u(w+y-s)+\tilde{V}(s)\}$ is the post-bargain utility, given the initial wealth $w$ and the current-period income $y$. Note that the outside option of each person is not to produce, possibly to consume a stored apple, and to get rematched with a different partner the next day. The Nash bargaining solution can be viewed as the equilibirum of a variation of the alternating-offers bargaining game in Binmore, Rubinstein, and Wolinsky (1986). Suppose that when two people are matched, there is a match-specific opportunity to produce, which disappears at a constant rate $q$ if agreement is not reached: If $t$ units of time have passed without agreement, the probability the opportunity will remain is $e^{-q t}$. Further, if $T$ units of time pass without agreement, the opportunity disappears altogether and each person receives no income in the period. Suppose that each round of offers takes $\tau$ units of time, so that the maximum number of rounds is $N=T / \tau$. In the limit of $N$ approaching infinity and $\tau$ approaching zero, holding $T$ and $q$, the equilibrium division of output is equivalent to the Nash bargaining solution.

An equilibrium is the wealth distribution function $\lambda(w)$, the value functions, $V(w)$, $\tilde{V}(w)$, and $W(w+y)$, and the income-probability function, $\pi\left(y ; w, w^{\prime}\right)$, that together satisfy (1) to (4).

\subsection{The Benchmark: Equal Sharing of Output}

Suppose that, instead of (4), each partner takes one apple for sure and takes the third apple with probability one half regardless of the partners' wealth levels: $\pi\left(1 ; w, w^{\prime}\right)=$ 
$\pi\left(2 ; w, w^{\prime}\right)=1 / 2$ for all $w$ and $w^{\prime}$. Since today's saving decision has no effect on the future income stream, the individual consumption/saving decision problem simplifies to maximizing utility, given the exogenous stochastic income process. Note that each person's income will be at least one apple at all times; the utility is linear beyond one apple of consumption; and $\beta(1-\delta)<1$. Then, there is no reason to save any apples.

Proposition 1: Under equal sharing of output (i.e., $\pi\left(1 ; w, w^{\prime}\right)=\pi\left(2 ; w, w^{\prime}\right)=1 / 2$ for all $w$ and $w^{\prime}$ ), everyone consumes all of the apples earned during the day and stores none.

Proof: See Appendix.

The proposition shows that any saving in this model is due to the effect of wealth on the sharing of output. Further, since $\beta(1-\delta)<1$, any saving is inefficient in that the aggregate utility (i.e., the sum of discounted utility across all people) is maximized when each person consumes at least one apple and all apples are consumed in each period. The welfare impact of implementing a policy of no saving will be detailed in Section 2.3.

\subsection{The Equilibrium: Unequal Sharing of Output}

Below I characterize the equilibrium. I consider three ranges of $\tilde{V}(1)-\tilde{V}(0)$ and derive the conditions on the parameter values that lead to each range in equilibrium.

Consume-All Equilibrium: $\tilde{V}(1)-\tilde{V}(0)<1$

If $\tilde{V}(1)-\tilde{V}(0)<1$, there will be no saving: $s=0$ for all $w$ and $y$. Further, $\lambda(0)=1$. We have $\pi(1 ; w, w)=\pi(2 ; w, w)=1 / 2$ for all $w$; and $W(w+y)=w+y+\tilde{V}(0)$ for all $w+y$. For the case of $\left(w, w^{\prime}\right)=(1,0)$, I can take the first-order condition in (4) and show that there are two possible solutions to the maximization problem. One solution is for each 
partner to take one apple each and for one of the two partners to take the third apple probabilistically: $\pi(1 ; 1,0)+\pi(2 ; 1,0)=1$ with the probability given by

$$
\tilde{\pi} \equiv \pi(2 ; 1,0)=\min \left\{1, \frac{1+\bar{u}}{2}\right\}
$$

Intuitively, the poor partner (i.e., the partner with $w=0$ ) has a disadvantage in bargaining since his outside option carries the starvation utility while the outside option of the rich partner (i.e., the partner with $w=1$ ) does not. From (1) and (5), we can express $\tilde{V}(1)-$ $\tilde{V}(0)$ in terms of $\tilde{\pi}$, and show that $\tilde{V}(1)-\tilde{V}(0)<1$ iff

$$
\tilde{\pi}<\frac{1}{\beta(1-\delta)}-\frac{1}{2}
$$

Equation (6) sets the upper limit to the rich person's income so that the incentive to store an apple is sufficiently small. From (5) and (6), we have:

$$
\min \left\{1, \frac{1+\bar{u}}{2}\right\}<\frac{1}{\beta(1-\delta)}-\frac{1}{2}
$$

The other solution to the maximization problem in (4) is for the poor partner to take one apple and for one of the two partners to take remaining two apples probabilistically: $\pi(0 ; 1,0)+\pi(2 ; 1,0)=1$. This changes the expressions on the righthand sides of (5) and (6), but we obtain the same parameter restriction (7). Intuitively, once the poor partner consumes one apple, the marginal utility of consuming a remaining apple is constant, so the two variations of the lottery deliver the same expected utility to each partner and do not affect the equilibrium conditions.

There are no solutions to (4) other than the above two. In particular, it is not a solution for the rich partner (i.e., $w=1$ ) to take two apples and for one of the two partners to take the remaining one apple probabilistically. Given the starvation utility, such an allocation can always be improved on (in terms of maximizing the Nash product) by guaranteeing one apple to the poor partner. In summary, we have: 
Proposition 2: The consume-all equilibrium (i.e., $\tilde{V}(1)-\tilde{V}(0)<1)$ exists if $(7)$ holds. In the consume-all equilibrium, the expected share of output is equal across partners in each match and everyone consumes all of the apples earned during the day and stores none. In the match of a poor person (i.e., a person with no stored apple) and a hypothetical rich person (i.e., a person who has deviated from the equilibrium path by storing an apple), the expected share of the rich partner's output is greater than that of the poor partner.

Proof: See Appendix.

In (5), (6), and (7), the starvation disutility $\bar{u}$ matters as a condition for the consume-all equilibrium by affecting the bargaining outcome of taking the third apple. As $\bar{u}$ rises, the probability of the rich partner taking the third apple rises, which raises the incentive to store an apple. On the other hand, a lower time discount rate $\beta$ or a higher depreciation probability $\delta$ lower the incentive to store an apple. Thus the consume-all equilibrium requires the combination of a low enough starvation utility $\bar{u}$, a low enough time discount rate $\beta$, and a high enough depreciation probability $\delta$.

Consume-Then-Save Equilibrium: $1<\tilde{V}(1)-\tilde{V}(0)<1+\bar{u}$

If $1<\tilde{V}(1)-\tilde{V}(0)<1+\bar{u}$, each person will save one apple if there any left after consuming one apple: $s=0$ if $w+y \leq 1$; and $s=1$ if $w+y \geq 2$. We have $\pi(1 ; w, w)=\pi(2 ; w, w)=1 / 2$ for all $w ; W(0)=-\bar{u}+\tilde{V}(0) ; W(1)=1+\tilde{V}(0)$; and $W(w+y)=w+y-1+\tilde{V}(1)$ for all $w+y \geq 2$. Taking the first-order condition in (4), I can show that $\{\pi(y ; 1,0)\}_{y}$ is summarized as: $\pi(1 ; 1,0)+\pi(2 ; 1,0)=1$ and

$$
\tilde{\pi} \equiv \pi(2 ; 1,0)=\min \left\{1, \frac{1}{2} \cdot\left(1-\tilde{V}(1)+\tilde{V}(0)+\frac{1+\bar{u}}{\tilde{V}(1)-\tilde{V}(0)}\right)\right\} .
$$

We have:

$$
V(0)=\left(\frac{1-\tilde{\lambda}}{2}+\tilde{\lambda} \cdot \tilde{\pi}\right)(1+\tilde{V}(0))+\left(\frac{1-\tilde{\lambda}}{2}+\tilde{\lambda} \cdot(1-\tilde{\pi})\right)(1+\tilde{V}(1))
$$




$$
V(1)=\left((1-\tilde{\lambda}) \cdot(1-\tilde{\pi})+\frac{\tilde{\lambda}}{2}\right)(1+\tilde{V}(1))+\left((1-\tilde{\lambda}) \cdot \tilde{\pi}+\frac{\tilde{\lambda}}{2}\right)(2+\tilde{V}(1))
$$

where $\tilde{\lambda} \equiv \lambda(1)$. The expressions inside large brackets are the transition probabilities, determined by the probability of being matched with a rich or a poor partner and the probability of taking the third apple conditional on being matched with a rich or a poor partner. Since the equilibrium is assumed to be a steady state, tomorrow's rich people are today's rich people each of whom saves an apple, plus the subset of today's poor people each of whom saves an apple, discounted by the depreciation probability:

$$
\tilde{\lambda} \equiv \lambda(1)=(1-\delta)\left(\tilde{\lambda}+(1-\tilde{\lambda})\left(\frac{1-\tilde{\lambda}}{2}+\tilde{\lambda}(1-\tilde{\pi})\right)\right)
$$

Given the parameters, $\beta, \delta$, and $\bar{u}$, we can use equations (2), (3), (8), (9), (10), and (11) to derive the equilibrium values of $\tilde{\pi}, \tilde{\lambda}$, and the value functions.

In order to find the conditions under which the consume-then-save equilibrium exists, consider the case of some depreciation: $\delta>0$. With the possibility of losing the stored apple over night, there will be some poor people in equilibrium: $\tilde{\lambda}<1$. Then, (11) can be rewritten as:

$$
\tilde{\pi}=\frac{1}{2}+\frac{1}{2 \tilde{\lambda}}-\frac{\delta}{1-\delta} \cdot \frac{1}{1-\tilde{\lambda}}
$$

Equation (12) implies a negative association between $\tilde{\pi}$ and $\tilde{\lambda}$. From (2), (3), (9), (10), and (12), I can derive:

$$
\tilde{V}(1)-\tilde{V}(0)=\frac{\beta((1-\delta) /(2 \tilde{\lambda})-\delta)}{1-\beta+\beta \delta /(1-\tilde{\lambda})}
$$

Equation (13) implies a negative association between $\tilde{\lambda}$ and $\tilde{V}(1)-\tilde{V}(0)$. Thus (12) and (13) can be interpreted as follows. If the probability of the rich partner taking the third apple, $\tilde{\pi}$, were to rise exogenously, the chance of a poor person to become rich declines, which leads to a reduced fraction of rich people in the population, $\tilde{\lambda}$, and a larger benefit of wealth, $\tilde{V}(1)-\tilde{V}(0)$. In (13), observe that there is $\lambda_{0} \in(0,1)$ such that $\tilde{V}(1)-\tilde{V}(0)>1$ 
iff $\lambda<\lambda_{0}$. Solving for $\lambda_{0}$ in (13) and then substituting the expression of $\lambda_{0}$ in (12), we have

$$
\tilde{\pi}>\pi_{0} \equiv \frac{1}{\beta(1-\delta)}-\frac{1}{2}
$$

iff $\lambda<\lambda_{0}$.

Now consider the limiting case of no depreciation: $\delta=0$. In (11), observe that with no depreciation of stored apples, a rich person will never become poor so that there will only be the rich people in equilibrium: $\tilde{\lambda}=1$ regardless of $\tilde{\pi}$. We can still show that $\tilde{V}(1)-\tilde{V}(0)>1$ iff $(14)$ holds with $\delta=0$.

Now, we can derive the conditions on the parameters for the consume-then-save equilibrium. Since $\tilde{\pi} \leq 1$, in (14) a necessary condition for an equilibrium with $\tilde{V}(1)-\tilde{V}(0)>1$ is:

$$
\frac{1}{\beta(1-\delta)}<\frac{3}{2}
$$

In order to have $\tilde{\pi}>\pi_{0}$ and $\tilde{V}(1)-\tilde{V}(0)>1$, in $(8)$, we need $\pi_{0}<(1+\bar{u}) / 2$ or:

$$
\bar{u}>u_{0} \equiv 2\left(\frac{1}{\beta(1-\delta)}-1\right)
$$

In summary, we have:

Proposition 3: The consume-then-save equilibrium (i.e., $1<\tilde{V}(1)-\tilde{V}(0)<1+\bar{u}$ ) exists if (15) and (16) hold. In the consume-then-save equilibrium, each partner takes at least one apple in any match, and each person stores one apple if there are any left after consuming one apple. In the match of a poor person (i.e., a person with no stored apple) and a rich person (i.e., a person with a stored apple), the probability of the rich person taking the third apple is higher than one half.

Proof: See Appendix.

Note that (15) and (16) are the opposite of (7). Thus the consume-then-save equilibrium requires the opposite of the condition for the consume-all equilibrium: the combination 
of a high enough starvation disutility $\bar{u}$, a high enough time discount rate $\beta$, and a low enough depreciation probability $\delta$, all of which raise the incentive to store an apple.

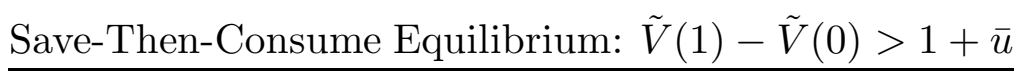

If $\tilde{V}(1)-\tilde{V}(0)>1+\bar{u}$, each person will save one apple if there any apples, and then consume what remains: $s=0$ if $w+y=0$; and $s=1$ if $w+y \geq 1$. I can show that this save-then-consume equilibrium does not exist.

Proposition 4: The save-then-consume equilibrium (i.e., $\tilde{V}(1)-\tilde{V}(0)>1+\bar{u}$ ), in which each person stores one apple if there are any apples, and then consumes any remaining apples, does not exist.

Proof: See Appendix.

The intuition is that the incentive to store an apple in this economy is to avoid starvation in case there is no income tomorrow, thereby raising the bargaining position tomorrow. Note that the equilibrium is implicitly assumed to be stationary, i.e., the saving decision depends only on current income and wealth. Then, the condition $\tilde{V}(1)-\tilde{V}(0)>1+\bar{u}$ implies that a person stores an apple even at the cost of starving only to ensure that he continue storing that apple tomorrow. By induction, storing an apple is to ensure storing that apple forever. It is hard to imagine why such a behavior should strengthen the bargaining position of the person.

The above three cases of $\tilde{V}(1)-\tilde{V}(0)$ leaves two borderline cases, $\tilde{V}(1)-\tilde{V}(0)=1$ and $\tilde{V}(1)-\tilde{V}(0)=1+\bar{u}$. First, consider the borderline case of $\tilde{V}(1)-\tilde{V}(0)=1$ : The incentive to store an apple is at the threshold so that each person is indifferent between consuming or storing an apple after having consuming one apple. Suppose that each person stores an apple with probability $\phi$ if there are any after consuming one. We can 
show that such an equilibrium, call it the consume-then-save-randomly equilibrium, exists if (7) holds with equality. In the consume-then-save-randomly equilibrium, the probability of taking the third apple $\tilde{\pi}$ is the same as under the consume-all or the consume-thensave equilibrium (i.e., $\tilde{\pi}$ given by (5) or by setting $\tilde{V}(1)-\tilde{V}(0)=1$ in (8)). Further, depending on $\phi$, the fraction of rich people $\tilde{\lambda}$ can take on any value between the value under the consume-all equilibrium, equal to zero, and the value under the consume-thensave equilibrium, given by (11). Thus, the consume-then-save-randomly equilibrium can be viewed as a convex combination of the consume-all and the consume-then-save equilibria with $\phi=0$ corresponding to the consume-all equilibrium and $\phi=1$ to the consume-thensave equilibrium.

Now consider the other borderline case of $\tilde{V}(1)-\tilde{V}(0)=1+\bar{u}$ : Each person is indifferent between consuming an apple to avoid starvation and storing it despite the starvation. Suppose that each person stores an apple with probability $\phi$ in such a situation. Using a variation of the proof of Lemma 3, we can show that such an equilibrium, call it save-randomly-then-consume equilibrium, does not exist under any parameter values.

Figure 1: Equilibrium Zones

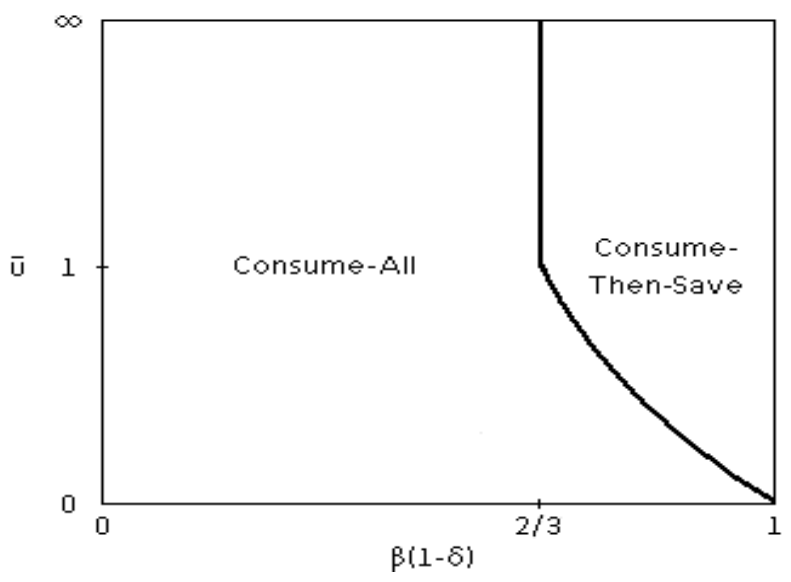

The various cases of $\tilde{V}(1)-\tilde{V}(0)$ considered above show that the space of the parameters, $\beta, \delta$, and $\bar{u}$, is divided into two zones, the zone of the consume-all equilibrium and 
the zone of the consume-then-save equilibrium with the threshold given by (7). Figure 1 visualizes the two zones. If (7) holds with equality, the incentive to store an apple is at the threshold (i.e., $\tilde{V}(1)-\tilde{V}(0)=1$ ) and the consume-then-save-randomly equilibria, the convex combinations of the consume-all and the consume-then-save equilibria, exist.

Consider the comparative statics of raising the value of $\bar{u}$ starting from (the epsilon above) zero, holding $\beta$ and $\delta$. Initially, we are at the consume-all equilibrium zone. Recall that there two possible lotteries, which deliver the same expected shares of output, in dividing the output in a match between a poor person and a rich person (who is on an off-equilibrium path since there are no rich people in equilibrium). Focus on the lottery in which only the third apple is divided probabilistically in order to compare with the consume-then-save equilibrium. Both the probability of a rich person taking the third apple, $\tilde{\pi}$, and the benefit of wealth, $\tilde{V}(1)-\tilde{V}(0)$, rise as $\bar{u}$ rises. If $\beta(1-\delta)$ is small enough so that (15) does not hold, $\tilde{\pi}$ reaches one eventually and a further rise in $\bar{u}$ does not affect the equilibrium. If $\beta(1-\delta)$ is large enough so that (15) holds, we would cross the threshold value $\bar{u}_{0}$ and switch to a consume-then-save equilibrium. Then, there will suddenly be some rich people in the economy $\left(\tilde{\lambda}=\lambda_{0}>0\right)$. The probability of a rich person taking the third apple, $\tilde{\pi}$, is equal to $\tilde{\pi}_{0}$ when $\bar{u}=\bar{u}_{0}$, continues to rise as $\bar{u}$ rises, and reaches one at some value $\bar{u}_{1}$. Similarly, the benefit of wealth, $\tilde{V}(1)-\tilde{V}(0)$, continues to rise as $\bar{u}$ rises, and reaches the maximum value when $\bar{u}=\bar{u}_{1}$. Meantime, the fraction

of rich people $\tilde{\lambda}$ declines from $\lambda_{0}$ and reaches some minimum value $\lambda_{1}$ when $\bar{u}$ reaches $\bar{u}_{1}$. Thereafter, a further rise of $\bar{u}$ does not affect the equilibrium.

\subsection{Welfare and Policy Implication}

Imagine the moment of making the consumption/saving decision (after the individual income has been realized) under the consume-then-save equilibrium, and consider introducing a (surprise) policy that induces people not to store any apples and to share output equally 
in each match from the next period, as in the consume-all equilibrium. One such policy is a wealth tax: Each stored apple is taxed with the probability $\varphi$. In Figure 1, there is a threshold $\hat{\delta}>\delta$ so that the equilibrium transits from a consume-then-save to a consume-all equilibrium as $\delta$ rises and crosses $\hat{\delta}$. In order to induce no saving, it is sufficient that the tax probability $\varphi$ satisfy: $(1-\varphi)(1-\delta)<(1-\hat{\delta})$. In equilibrium, no wealth taxes would be collected since nobody would store an apple. An alternative policy is (the announcement of) a progressive income tax/subsidy, for instance, a policy that subsidizes one apple to a person with no income, and taxes a person with an income of three apples one apple. The subsidy would eliminate the bargaining advantage of a rich person, so nobody would store an apple, and everyone's expected income after taxes and subsidies would be $3 / 2$ apples as in the consume-all equilibrium. There may be other policies that would lead to the same outcome. Below, I will refer to any of these policies that induce no saving and the equal sharing of output as the no-saving policy.

In order to assess the welfare impact of the no-saving policy, first consider a would-berich person (i.e., a person who would have stored an apple under the consume-then-save equilibrium). From (2), (3), (9), and (10), the expected discounted utility of such a person under the consume-then-save equilibrium is:

$$
\tilde{V}_{s}(1)=\frac{\beta}{1-\beta} \cdot\left(1+(1-\tilde{\lambda}) \cdot \tilde{\pi}+\frac{\tilde{\lambda}}{2}-\frac{\delta}{\beta(1-\delta)} \cdot\left(\tilde{V}_{s}(1)-\tilde{V}_{s}(0)\right)\right)
$$

where the subscript $s$ denotes the consume-then-save equilibrium. Under the no-saving policy, the expected discounted utility of the above would-be-rich person is the marginal utility of consuming a second or a third apple (instead of storing it), which is equal to one, plus the expected discounted utility under the equal sharing of output with no saving from the next period, which is equal to:

$$
\tilde{V}_{c}(0)=\frac{3}{2} \cdot \frac{\beta}{1-\beta}
$$


where the subscript $c$ denotes the consume-all equilibrium. Let $\Omega$ denote the utility gain (loss) of the would-be-rich person upon the implementation of the no-saving policy:

$$
\Omega \equiv 1+\tilde{V}_{c}(0)-\tilde{V}_{s}(1)
$$

If the depreciation probability $\delta=0$, the fraction of rich people $\tilde{\lambda}=1$ under the consumethen-save equilibrium so that $\tilde{V}_{s}(1)=3 / 2 \cdot \beta /(1-\beta)$ in (17) and $\Omega=1>0$ in (18). If $\delta>0$, we can express $\tilde{V}_{s}(1)$ in terms of $\beta, \delta$, and $\tilde{\lambda}$ only, using (12) and (13). Given $\beta$ and $\delta$, the remaining parameter $\bar{u}$ determines $\tilde{\lambda}$ in the range $\left[\lambda_{1}, \lambda_{0}\right]$, with a higher $\bar{u}$ leading to a lower $\tilde{\lambda}$, as discussed in Section 2.2. We can show that $\partial \tilde{V}_{s}(1) / \partial \tilde{\lambda}<0$ within the range of $\left[\lambda_{1}, \lambda_{0}\right]$, which implies that $\tilde{V}_{s}(1)$ takes on the highest value when $\bar{u} \geq \bar{u}_{1}$ so that $\tilde{\lambda}=\lambda_{1}$. An interpretation is that as $\bar{u}$ rises, the one-period income gain of a rich person (i.e., $1+(1-\tilde{\lambda}) \cdot \tilde{\pi}$ in $(17))$ rises. The annuity value of the utility loss from eventually losing the stored apple (i.e., $\delta /(\beta(1-\delta)) \cdot\left(\tilde{V}_{s}(1)-\tilde{V}_{s}(0)\right)$ in $\left.(17)\right)$ also rises but this is a second-order effect as it is due to the rise of the one-period income gain. Let $\Omega_{1}$ denote the value of $\Omega$ when $\bar{u} \geq \bar{u}_{1}$ given $\beta$ and $\delta$. Then, $\Omega \geq \Omega_{1}$. We can show that, somewhat surprisingly, $\Omega_{1}>0$ for any $\beta$ and $\delta$ that satisfy (15). Then, $\Omega \geq \Omega_{1}>0$ for any $\beta, \delta$, and $\bar{u}$ under which the consume-then-save equilibrium exists. Therefore, a would-be-rich person becomes better off upon the implementation of the no-saving policy.

Now, consider a would-be-poor person (i.e., a person who would not have stored an apple under the consume-then-save equilibrium). The expected discounted utility of such a person under the consume-then-save equilibrium is $\tilde{V}_{s}(0)$. The expected discounted utility of the person under the no-saving policy is $\tilde{V}_{c}(0)$. Since $\tilde{V}_{s}(1)-\tilde{V}_{s}(0)>1$, we have:

$$
\tilde{V}_{s}(0)<\tilde{V}_{s}(1)-1=\tilde{V}_{c}(0)-\Omega<\tilde{V}_{c}(0) .
$$

Therefore, a would-be-poor people also becomes better off under the no-saving policy. In summary, we have: 
Proposition 5: Suppose that the economy is at a moment of making the consumption/saving decision under the consume-then-save equilibrium, and that a policy that induces no saving and the equal sharing of output is implemented unexpectedly. Then, everyone in the economy becomes better off.

Proof: See Appendix.

Proposition 5 starkly shows the inefficiency of saving in this model. Recall that the only motive for saving is to obtain a greater share of output tomorrow. Since the total output is fixed, the advantage of wealth in bargaining is at the disadvantage of other people. Storing an apple enables a person to receive the third apple with the probability of more than one half when matched with a poor partner; it also enables the person to avoid receiving the third apple with the probability of less than one half when matched with a rich partner. Thus, storing an apple has a negative externality on both the poor and the rich people. Consider the limiting case of no depreciation $(\delta=0)$ to focus on the externality on the rich people. With $\delta=0$, there are only rich people $(\tilde{\lambda}=1)$ and everyone receives the third apple with the probability of one half in equilibrium. If everyone stops storing apples, each person can consume one extra apple immediately and continue receiving the third apple with the probability of one half from tomorrow. Therefore, everyone becomes better off under the no-saving policy. Proposition 5 shows that the force of the externality is strong enough so that everyone becomes better off whenever the economy is in a consume-thensave equilibrium.

\section{Extensions}

In the previous section, I made a number of simplifying assumptions in order to make the logic of the inefficiency of wealth accumulation clear. A notable assumption is that matching is random. On the contrary, suppose that the poor are matched only with 
the poor and the rich are matched only with the rich. Then the output would be equally divided in all matches, eliminating the incentive to accumulate wealth. An interpretation of random matching is that a production opportunity arises for two randomly chosen people. Random matching can be rephrased as the assumption that the production opportunities arise for people of diverse wealth levels at least sometimes.

In the remainder of this section, I address two other assumptions, indivisibility of output and the limited storage capacity. I relax these assumptions and derive the conditions under which the equilibrium with a wealth accumulation exists.

\subsection{Divisibility of Output}

Suppose that each apple is divisible, so that dividing the output by a fraction of an apple and storing a fraction of an apple are possible. The one-period utility is $E[U(c)]$ where $U(c)=c \cdot I(c \geq 1)+(c-(1-c) \cdot \bar{u}) \cdot I(c<1)$, where $c$ is assumed to be any nonnegative value. This utility function is an expansion of the utility function in Section 2, interpolating the utility values when $c$ is not an integer. The one-period budget constraint is $c+s=w+y$, where $s, w \in[0,1]$.

I will focus on the limiting case of no depreciation of stored apples (i.e., $\delta=0$ ). Otherwise, the model environment is the same as in Section 2. I will construct an equilibrium where everyone maintains one stored apple (i.e., $\tilde{\lambda}=1$ ) as in the consume-then-save equilibrium with no depreciation. In order to insure that there is no incentive for a person to deviate from the equilibrium by saving less than one apple, I will characterize the offequilibrium path (i.e., the bargaining outcome and the consumption/saving pattern of a person with less than one saved apple).

The utility of a person with wealth $w$ at the beginning of a period is:

$$
V(w)=\sum_{y} \pi(y ; w, 1) \max _{s \in \Upsilon(w+y)}\{u(w+y-s)+\beta V(s)\}
$$


where $w \in[0,1]$ and $\Upsilon(w+y) \equiv[0, \min \{1, w+y\}]$. The bargaining outcome maximizes the Nash product: for each $w,\{\pi(y ; w, 1)\}_{y}$ solves

$$
\max \left\{\left(\sum_{y} \pi(y ; w, 1) \cdot W(w+y)-W(w)\right)\left(\sum_{y} \pi(y ; w, 1) \cdot W(4-y)-W(1)\right)\right\}
$$

where $W(w+y) \equiv \max _{s \in \Upsilon(w+y)}\{u(w+y-s)+\beta V(s)\}$. An equilibrium is the value functions, $V(w)$ and $W(w+y)$, and the income-probability function, $\pi(y ; w, 1)$, that together satisfy $(1)^{\prime}$ and $(4)^{\prime}$.

The conjectured equilibrium properties are:

$$
1 \leq \beta(V(1)-V(0)) \leq 1+\bar{u}
$$

and

$$
\beta(V(w)-V(0)) \leq w \cdot \beta(V(1)-V(0))
$$

for all $w \in[0,1]$. Equation (20) implies an incentive to store an apple after consuming one. Equation (21) implies that everyone prefers a fair lottery of storing a whole apple or none to the certainty of storing a fraction of an apple. Both of these properties mimic the consume-then-save equilibrium in Section 2.

Using the consumption/saving pattern given by (20) and(21), we can deduce the pattern of $W(w)$ and then, drawing the utility frontiers under the feasible division of output, deduce the pattern of the bargaining outcome. Figure 2 illustrates the expected value of the sum of wealth and income, $w+\sum_{y} y \pi(y ; w, 1)$, as a function of wealth $w$. If a person's wealth is below $\tilde{w}_{1}$, his wealth has no value since his income will be just sufficient to avoid starvation regardless of his wealth. In maximizing $(4)^{\prime}$, the solution is at a corner. If a person's wealth is between $\tilde{w}_{1}$ and $\tilde{w}_{2}$, his income is enough to consume an apple and to save an apple with some probability. Thus obtaining an apple beyond starvation is (endogenously) probabilistic reminiscent to the model in Section 2. If a person's wealth is between $\tilde{w}_{2}$ and $\tilde{w}_{3}$, his wealth is enough to consume an apple and to save an apple with 
certainty. In maximizing $(4)^{\prime}$, the solution is at another corner. If a person's wealth is above $\tilde{w}_{3}$, his wealth is enough to consume more than an apple and to save an apple with certainty.

Figure 2: Expected Sum of Wealth and Income as a Function of Wealth

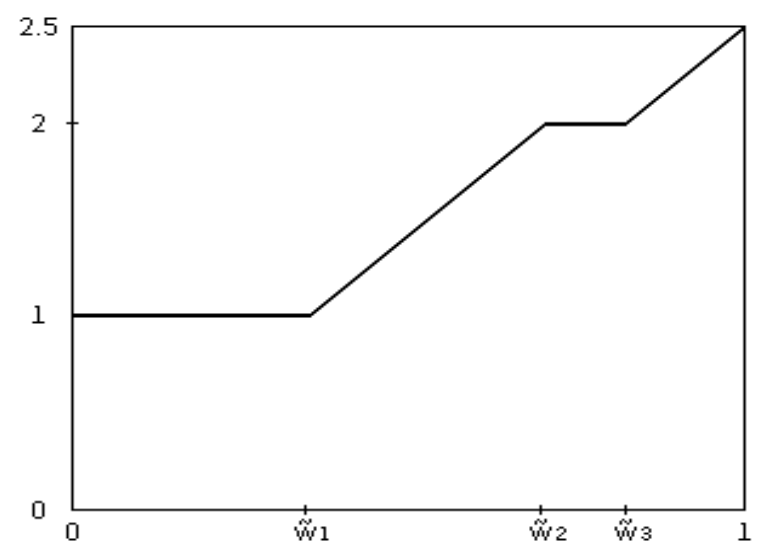

Using the pattern of the bargaining outcome, we can further deduce the pattern of the value function $V(w)$, illustrated in Figure 3 .

Figure 3: Expected Utility as a Function of Wealth

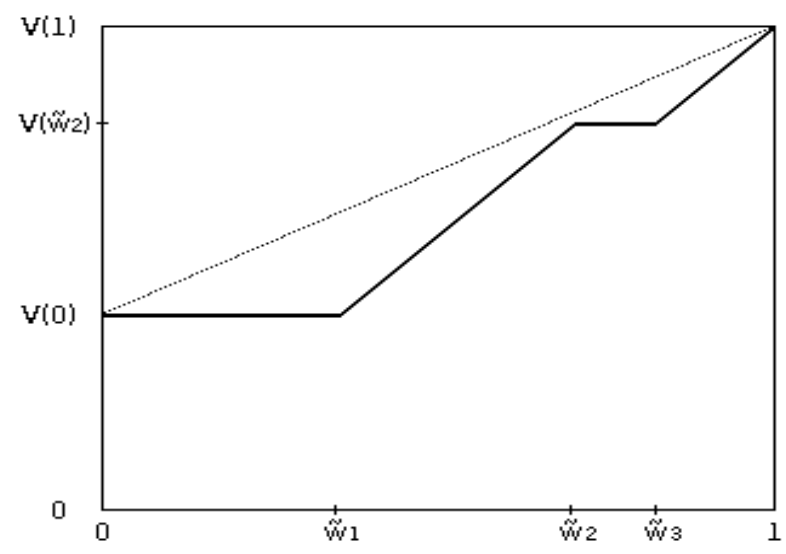

In Figure 3, the dotted line is the righthand side of (21), multiplied by $1 / \beta$. In order for $(21)$ to be satisfied, the value function $V(w)$ must lie below the dotted line for all 
$w \in(0,1)$. Observe that this is equivalent to $V\left(\tilde{w}_{2}\right)$ lying below the dotted line. Using this observation, we can show that (21) is equivalent to:

$$
1+\bar{u} \geq \frac{\beta}{2(1-\beta)}\left(\frac{\beta}{2(1-\beta)^{2}}-1\right)
$$

Further, given (22), (20) holds iff:

$$
\beta \geq \frac{2}{3}
$$

Equation $(15)^{\prime}$ is equivalent to (15) for the case of $\delta=0$ while (22) is more restrictive than (16). In summary, we have:

Proposition 6: An equilibrium with $\lambda(1)=1$ exists if $(15)^{\prime}$ and (22) hold.

Proof: See Appendix.

Thus the equilibrium in which everyone maintains one stored apple (i.e., $\lambda(1)=1$ ), as in the consume-then-save equilibrium with $\delta=0$ in Section 2, exists if the valuation of future consumption is high enough (i.e., $\beta$ large enough) and the starvation disutility is high enough (i.e., $\bar{u}$ large enough). The threshold disutility is higher than in Section 2.

We can conjecture possible equilibria when the starvation disutility $\bar{u}$ is below the threshold in (22). In Figure 3, the value function would rise above the dotted line. The bargaining outcome would then feature the probabilistic allocation of a fraction of an apple (instead of a whole apple) to a partner with additional flat segments in Figure 2, and the consumption/saving behavior would feature storing a fraction of an apple. Regardless of these complications in the off-equilibrium paths, the equilibrium with $\lambda(1)=1$ may very well exist for a segment of $\bar{u}$ below the threshold in (22). In this sense, the characterization of the equilibrium in this section is for the special case of non-fractional saving, mimicking the equilibrium in Section 2. 


\subsection{Unlimited Storage Capacity}

Now suppose that the storage capacity is unlimited so that the wealth $w$ and the saving $s$ can take on any non-negative integers $\{0,1,2, \ldots\}$. As in Section 3.1, assume that there is no depreciation of stored apples (i.e., $\delta=0$ ). Otherwise, maintain the same environment as in Section 2. I will construct an equilibrium where everyone maintains a fixed number of stored apples, i.e., $\lambda(\bar{w})=1$ for some $\bar{w} \geq 1$. In order to insure that there is no incentive for a person to deviate from the equilibrium, I will characterize the off-equilibrium path. (i.e., the bargaining outcome and the consumption/saving pattern of a person with more or less than $\bar{w}$ stored apples).

The utility of a person with wealth $w$ at the beginning of a period is:

$$
V(w)=\sum_{y} \pi(y ; w, \bar{w}) \max _{s \in \Upsilon(w+y)}\{u(w+y-s)+\beta V(s)\}
$$

where $w \in\{0,1, \ldots\}$ and $\Upsilon(w+y) \equiv\{0,1, \ldots, w+y\}$. The bargaining outcome maximizes the Nash product: for each $w,\{\pi(y ; w, \bar{w})\}_{y}$ solves

$$
\max \left\{\left(\sum_{y} \pi(y ; w, \bar{w}) W(w+y)-W(w)\right)\left(\sum_{y} \pi(y ; w, \bar{w}) \cdot W(\bar{w}+3-y)-W(\bar{w})\right)\right\}
$$

where $W(w+y) \equiv \max _{s \in \Upsilon(w+y)}\{u(w+y-s)+\tilde{V}(s)\}$. An equilibrium is the wealth level $\bar{w}$, the value functions, $V(w)$ and $W(w+y)$, and the income-probability function, $\pi(y ; w, \bar{w})$, that together satisfy $(1)^{\prime \prime}$ and $(4)^{\prime \prime}$.

The conjectured equilibrium properties are: $\lambda(\bar{w})=1$ for some $\bar{w} \geq 1$;

$$
1 \leq \beta(V(\bar{w}-n)-V(\bar{w}-n-1)) \leq 1+\bar{u}
$$

for all $n \in\{0,1, \ldots, \bar{w}-1\}$;

$$
\beta(V(\bar{w}-n)-V(\bar{w}-n-1)) \leq \beta(V(\bar{w}-n-1)-V(\bar{w}-n-2))
$$


for all $n \in\{0,1, \ldots, \bar{w}-2\}$; and

$$
\beta(V(\bar{w}+n)-V(\bar{w}+n-1)) \leq 1
$$

for all $n \geq 1$. Equation (23) is a generalized version of the condition, $1<\tilde{V}(1)-\tilde{V}(0)<$ $1+\bar{u}$, for the consume-then-save equilibrium in Section 2 , and implies an incentive to save an apple after consuming one when $w<\bar{w}$. Equation (24) ensures that the bargaining outcome takes the form of the probabilistic gain/loss of the third apple (rather than the probabilistic gain/loss of multiple apples) so that a person with $w<\bar{w}$ probabilistically accumulates one apple at a time. Equation (25) implies no incentive to store an apple beyond $\bar{w}$.

Given (23), (24), and (25), we can express $V(w)$ in terms of $\pi(y ; w, \bar{w})$. Also, we can express $W(w)$ in terms of $V(w)$ and then, taking the first-order condition in $(4)^{\prime \prime}$, express $\pi(y ; w, \bar{w})$ in terms of $V(w)$. We can then show that the conditions $\beta(V(\bar{w})-V(\bar{w}-1))>1$ and $\beta(V(\bar{w}+1)-V(\bar{w}))<1$ in $(23)$ and $(25)$ are equivalent to:

$$
\frac{1}{\beta}-\frac{1}{2} \equiv \pi_{0} \leq \check{\pi}(\bar{w}-1) \leq \pi_{1} \equiv \min \left\{1, \frac{1}{\beta}-\frac{1}{2} \cdot \frac{\beta}{2-\beta}\right\}
$$

where $\check{\pi}(\bar{w}-n) \equiv \pi(1 ; \bar{w}-n, \bar{w})=\pi(2 ; \bar{w}, \bar{w}-n)$. In words, $\check{\pi}(\bar{w}-n)$ is the probability of a person with $\bar{w}$ taking the third apple in a match with a person with $\bar{w}-n$. The first inequality is a generalized version of (14) in Section 2: It is necessary for any equilibrium with $\bar{w} \geq 1$. The second inequality was absent in Section 2 , since a storage capacity was assumed in that section. Intuitively, a larger value of $\pi(2 ; \bar{w}, \bar{w}-1)$ implies a larger value of $\beta(V(\bar{w})-V(\bar{w}-1))$. The outside option of a person with $\bar{w}$ in bargaining with a person with $\bar{w}+1$ is negatively affected by a higher value of $\beta(V(\bar{w})-V(\bar{w}-1))$. Thus, a high enough value of $\beta(V(\bar{w})-V(\bar{w}-1))$ implies a small enough value of $\pi(2 ; \bar{w}, \bar{w}+1)$ and a large enough value of $\beta(V(\bar{w}+1)-V(\bar{w}))$. Since $\pi(2 ; \bar{w}, \bar{w}-1) \leq 1$, a necessary condition for the equilibrium with $\bar{w} \geq 1$ is:

$$
\beta \geq \frac{2}{3}
$$


which is an analog of (15) in Section 2.

In order to find the equilibrium values of $\bar{w}, \check{\pi}(w)$, and $\bar{u}$, we can first derive:

$$
\frac{\beta(1-\check{\pi}(\bar{w}-n))}{1-\beta \check{\pi}(\bar{w}-n-1)}=2 \check{\pi}(\bar{w}-n)-1+\frac{\beta}{2(1-\beta \check{\pi}(\bar{w}-1))}
$$

which yields $\check{\pi}(\bar{w}-n-1)$ given $\check{\pi}(\bar{w}-n)$. We can use (26) to derive the sequence of $(\check{\pi}(\bar{w}-1), \check{\pi}(\bar{w}-2), \ldots, \check{\pi}(\bar{w}-\bar{n}))$ starting from any value of $\check{\pi}(\bar{w}-1) \in\left[\pi_{0}, \pi_{1}\right]$. The value of $\check{\pi}(\bar{w}-n)$ rises in $n$ and there is a maximum value of $n$, call it $\bar{n}$, with $\check{\pi}(\bar{w}-\bar{n}) \leq 1$. Then, for any $n \in\{1,2, \ldots, \bar{n}\}$, we can set $\bar{w}=n$ so that $(\check{\pi}(0), \check{\pi}(1), \ldots, \check{\pi}(\bar{w}))=(\check{\pi}(\bar{w}-$ $n), \check{\pi}(\bar{w}-n+1), \ldots, \check{\pi}(\bar{w}))$. Given $\pi(0), \bar{u}$ is given by:

$$
\check{\pi}(0) \leq \frac{1}{2}\left(1-\beta(V(\bar{w})-V(\bar{w}-1))+\frac{1+\bar{u}}{\beta(V(1)-V(0))}\right)
$$

with the strict equality if $\check{\pi}(0)<1$. Inequality (27) is a generalized version of (8). This algorithm of finding the equilibrium can be understood intuitively as follows. The bargaining advantage of a person with the equilibrium wealth is greater when matched with a person with less wealth or, equivalently, a person with less wealth has a greater disadvantage in bargaining with a person with the equilibrium wealth. These bargaining (dis)advantages provide the incentive to accumulate wealth. Since the probability of taking the third apple is bounded by one, the bargaining (dis)advantage is bounded and there is a maximum wealth sustainable in equilibrium.

Analyzing the above algorithm of finding the equilibrium, we can derive some other equilibrium properties. Observe that equilibria vary along two dimensions, $\bar{w}$ and $\check{\pi}(\bar{w}-1)$. For $\bar{w}=1$, any $\check{\pi}(\bar{w}-1)=\check{\pi}(0) \in\left[\pi_{0}, \pi_{1}\right]$ is supported as an equilibrium by some value of $\bar{u}$. As $\bar{w}$ rises, the equilibrium values of $\check{\pi}(\bar{w}-1)$ may be truncated from the above, since the sequence of $\check{\pi}(w)$ derived by (26) reaches the upper bound of one possibly sooner for a higher value of $\check{\pi}(\bar{w}-1) \in\left[\pi_{0}, \pi_{1}\right]$. There is a maximum equilibrium wealth, call it $\bar{\omega}$, so that for any $\bar{w}>\bar{\omega}$, there is no range of $\check{\pi}(\bar{w}-1)$ that supports $\bar{w}$ as an equilibrium. For any $\bar{w} \leq \bar{\omega}$, let $\pi_{2}(\bar{w}) \in\left[\pi_{0}, \pi_{1}\right]$ denote the cutoff value so that $\bar{w}$ and any 
$\check{\pi}(\bar{w}-1) \in\left[\pi_{0}, \pi_{2}(\bar{w})\right]$ are supported as an equilibrium by some value of $\bar{u}$. Equation (26) maps the range $\left[\pi_{0}, \pi_{2}(\bar{w})\right]$ into a range of $\check{\pi}(0),\left[\check{\pi}(0)\left|\left(\bar{w}, \pi_{0}\right), \check{\pi}(0)\right|\left(\bar{w}, \pi_{2}(\bar{w})\right)\right]$, where the cutoff values, $\check{\pi}(0) \mid\left(\bar{w}, \pi_{0}\right)$ and $\check{\pi}(0) \mid\left(\bar{w}, \pi_{2}(\bar{w})\right)$, are higher for a higher $\bar{w}$ as long as they are smaller than one. Further, $(27)$ maps the range $\left[\check{\pi}(0)\left|\left(\bar{w}, \pi_{0}\right), \check{\pi}(0)\right|\left(\bar{w}, \pi_{2}(\bar{w})\right)\right]$ into a finite range of $\bar{u},[\check{u}(\bar{w}), \hat{u}(\bar{w})]$, if $\check{\pi}(0) \mid\left(\bar{w}, \pi_{2}(\bar{w})<1\right.$, and into an infinite range of $\bar{u},[\check{u}(\bar{w}), \infty)$, if $\check{\pi}(0) \mid\left(\bar{w}, \pi_{2}(\bar{w})=1\right.$. The cutoff values, $\check{u}(\bar{w})$ and $\hat{u}(\bar{w})$, are higher for a higher $\bar{w}$, and if the range of $\bar{u}$ is infinite for some $\bar{w}$, it is infinite for a higher $\bar{w}$. In this sense, a greater starvation disutility $\bar{u}$ supports a higher equilibrium wealth level $\bar{w}$. Further, we can show that $\check{u}(\bar{w}+1)<\hat{u}(\bar{w})$ so that the ranges $\{[\check{u}(\bar{w}), \hat{u}(\bar{w})]\}_{\bar{w}}$, indexed by $\bar{w}$, overlap with each other. Thus, the ranges of $\bar{u}$ that support various values of $\bar{w}$ as equilibria form a single contiguous set. In summary, we have:

Proposition 7: If $(15)^{\prime}$ holds, there is $\bar{\omega} \geq 1$ such that, for any $\bar{w} \in\{1,2, \ldots, \bar{\omega}\}$, there is a range of $\bar{u}$, call it $\bar{U}(\bar{w})$, under which an equilibrium with $\lambda(\bar{w})=1$ exists. The range is either finite (i.e., $\bar{U}(\bar{w})=[\check{u}(\bar{w}), \hat{u}(\bar{w})]$ ) or infinite (i.e., $\bar{U}(\bar{w})=[\check{u}(\bar{w}), \infty))$. The range is increasing in $\bar{w}$ (i.e., $\check{u}(\bar{w})<\check{u}(\bar{w}+1) ; \hat{u}(\bar{w})<\hat{u}(\bar{w}+1)$; and $\bar{U}(\bar{w}+1)$ is infinite if $\bar{U}(\bar{w})$ is infinite). Further, the ranges are contiguous (i.e., $\check{u}(\bar{w}+1)<\hat{u}(\bar{w}))$ so that an equilibrium with some $\bar{w}$ exists if $\bar{U}(\bar{\omega})$ is finite and $\bar{u} \in[\check{u}(1), \hat{u}(\bar{\omega})]$, or if $\bar{U}(\bar{\omega})$ is infinite and $\bar{u} \in[\check{u}(1), \infty)$.

Proof: See Appendix.

We can show that, for the case of $\bar{w}=1$, the equilibrium range of $\bar{u}$ is:

$$
\bar{U}(1)= \begin{cases}{[\breve{u}(1), \hat{u}(1)]=\left[2 \cdot\left(\frac{1}{\beta}-1\right), \frac{8}{\beta} \cdot\left(\frac{1}{\beta}-1\right)\right]} & \text { if } \pi_{1}<1 \\ {[\check{u}(1), \infty)=\left[2 \cdot\left(\frac{1}{\beta}-1\right),\right.} & \text { if } \pi_{1}=1 .\end{cases}
$$

The value of $\check{u}(1)$ is the same cutoff value as in (16) in Section 2. The difference is that, under the unlimited storage capacity assumed in this section, the equilibrium range of $\bar{u}$ 
may be truncated from the above in order to ensure that there is no incentive to save more than one apple.

\section{Conclusion}

This paper presents a formal model of the bargaining motive of wealth accumulation and characterized its welfare properties. The main assumption is the starvation disutility, which weakens the bargaining position of a poor person and strengthens that of a rich person in an otherwise symmetric bargaining, providing the incentive to accumulate wealth. Since raising a person's advantage in bargaining is possible only by reducing the advantage of the others in the economy, wealth accumulation is inefficient. Policies such as a wealth tax and a progressive income tax can improve the welfare.

The model is stylized in order to make the bargaining motive of wealth accumulation and its welfare properties transparent. In reality, of course, there are other motives for accumulating wealth such as returns from investing in capital and precautionary saving. These other motives of wealth accumulation seem conceptually separated from the bargaining motive. Nonetheless, any convincing policy recommendations would need to balance the policy implications of various motives of wealth accumulation. 


\section{References}

Bills, M., Chang, Y., and Kim, S. (2011), "Worker Heterogeneity and Endogenous Separations in a Matching Model of Unemployment Fluctuations," American Economic Journal: Macroeconomics 3:128-154.

Binmore, K., Rubinstein, A., and Wolinsky, A. (1986), "The Nash Bargaining Solution in Economic Modeling," Rand Journal of Economics 17:176-188.

Cole, H., Mailath, G., and Postlewaite, A. (1992), "Social Norms, Savings Behavior, and Growth" Journal of Political Economy 100:1092-1125.

Corneo, G. and Jeanne, O. (1998), "Social Organization, Status, and Savings Behavior," Journal of Public Economics 70:37-51.

Frank, R. (1985), "Demand for Unobservable and Other Nonpositional Goods," American Economic Review 75:101-116.

Kihlstrom, R., Roth, A., and Schmeidler, D. (1981), "Risk Aversion and Solutions to Nash's Bargaining Problem" in Game Theory and Mathematical Economics, edited by Moeschlin, O. and Pallaschke, D., Amsterdam: North-Holland.

Konrad, K. (1992), "Wealth Seeking Reconsidered," Journal of Economics Behavior and Organization 75:101-116.

Krusell, P., Mukoyama, T., and Sahin, A. (2010), "Labour-Market Matching with Precautionary Savings and Aggregate Fluctuations," Review of Economic Studies 77:14771507. 


\section{Appendix: Proofs of Propositions}

\section{Proof of Proposition 1}

We have

$$
\begin{aligned}
& V(0)=\frac{1}{2} \max _{s \in\{0,1\}}\{U(1-s)+\tilde{V}(s)\}+\frac{1}{2} \max _{s \in\{0,1\}}\{U(2-s)+\tilde{V}(s)\} ; \\
& V(1)=\frac{1}{2} \max _{s \in\{0,1\}}\{U(2-s)+\tilde{V}(s)\}+\frac{1}{2} \max _{s \in\{0,1\}}\{U(3-s)+\tilde{V}(s)\} .
\end{aligned}
$$

Observe that

$$
V(0) \geq \frac{1}{2} \cdot(U(1)+\tilde{V}(0))+\frac{1}{2} \cdot(U(2)+\tilde{V}(0))
$$

so that

$$
\begin{aligned}
V(1)-V(0) & \leq \frac{1}{2} \max _{s \in\{0,1\}}\{U(2-s)-U(1)+\tilde{V}(s)-\tilde{V}(0)\} \\
& +\frac{1}{2} \max _{s \in\{0,1\}}\{U(3-s)-U(2)+\tilde{V}(s)-\tilde{V}(0)\} \\
& =\max \{1, \tilde{V}(1)-\tilde{V}(0)\} .
\end{aligned}
$$

Then,

$$
\tilde{V}(1)-\tilde{V}(0)=\beta(1-\delta)(V(1)-V(0)) \leq \beta(1-\delta) \max \{1, \tilde{V}(1)-\tilde{V}(0)\}
$$

so that

$$
\tilde{V}(1)-\tilde{V}(0) \leq \beta(1-\delta)<1 \text {. }
$$

This implies that $s=0$ and $c=w+y$ and for all $w$ and $y$.

\section{Proof of Proposition 2}

Consider first-order conditions when $\left(w, w^{\prime}\right)=(1,0)$ in (4). A first-order condition is $\pi(1 ; 1,0)+\pi(2 ; 1,0)=1$ and

$$
\begin{aligned}
& (W(3)-W(2))(\tilde{\pi} W(1)+(1-\tilde{\pi}) W(2)-W(0)) \\
+ & (W(1)-W(2))(\tilde{\pi} W(3)+(1-\tilde{\pi}) W(2)-W(1)) \geq 0
\end{aligned}
$$


with the strict equality if $\tilde{\pi} \equiv \pi(2 ; 1,0)<1$. Using that $W(w+y)=w+y+\tilde{V}(0)$ for all $w+y$, we can show that (A1) is equivalent to (5) in the main text. Noting that $\pi(1 ; 0,0)=\pi(2 ; 0,0)=1 / 2$ and $\lambda(0)=1$, we have:

$$
\begin{gathered}
V(0)=\frac{1}{2} \cdot(1+\tilde{V}(0))+\frac{1}{2} \cdot(2+\tilde{V}(0)) ; \\
V(1)=(1-\tilde{\pi}) \cdot(2+\tilde{V}(0))+\tilde{\pi} \cdot(3+\tilde{V}(0)) .
\end{gathered}
$$

Then,

$$
\tilde{V}(1)-\tilde{V}(0)=\beta(1-\delta)(V(1)-V(0))=\beta(1-\delta)\left(\frac{1}{2}+\tilde{\pi}\right)<1
$$

iff

$$
\tilde{\pi}<\frac{1}{\beta(1-\delta)}-\frac{1}{2}
$$

From (5) and (6), we have:

$$
\min \left\{1, \frac{1+\bar{u}}{2}\right\}<\frac{1}{\beta(1-\delta)}-\frac{1}{2}
$$

Another first-order condition in (4) with $\left(w, w^{\prime}\right)=(1,0)$ is: $\pi(0 ; 1,0)+\pi(2 ; 1,0)=1$ and

$$
\begin{aligned}
& (W(3)-W(1))(\tilde{\pi} W(1)+(1-\tilde{\pi}) W(2)-W(0)) \\
+ & (W(1)-W(2))(\tilde{\pi} W(3)+(1-\tilde{\pi}) W(1)-W(1)) \geq 0
\end{aligned}
$$

with the strict equality if $\tilde{\pi} \equiv \pi(2 ; 1,0)<1$. Repeating the above steps, we can show that (A2) is equivalent to

$$
\tilde{\pi} \equiv \pi(2 ; 1,0)=\min \left\{1, \frac{3+\bar{u}}{4}\right\}
$$

and $\tilde{V}(1)-\tilde{V}(0)<1$ iff

$$
\tilde{\pi}<\frac{1}{2 \beta(1-\delta)}+\frac{1}{4} .
$$

From (A3) and (A4), we also have (7). 
There are no first-order conditions, other than the above two, that yield additional solutions. In particular, consider the following first-order condition: $\pi(2 ; 1,0)+\pi(3 ; 1,0)=1$ and

$$
\begin{aligned}
& (W(4)-W(3))(\bar{\pi} W(0)+(1-\bar{\pi}) W(1)-W(0)) \\
+ & (W(0)-W(1))(\bar{\pi} W(4)+(1-\bar{\pi}) W(3)-W(1)) \leq 0
\end{aligned}
$$

with the strict equality if $\bar{\pi} \equiv \pi(3 ; 1,0)>0$. We can show that (A5) is equivalent to $\bar{\pi}=0$. Thus, only the corner solution is possible, but this only completes the corner solution in (5) and (A3). Therefore, we have Proposition 2.

\section{Proof of Proposition 3}

Consider first-order conditions when $\left(w, w^{\prime}\right)=(1,0)$ in (4). A first-order condition is $\pi(1 ; 1,0)+\pi(2 ; 1,0)=1$ and (A1) with the strict equality if $\tilde{\pi} \equiv \pi(2 ; 1,0)<1$. Using

that $W(0)=-\bar{u}+\tilde{V}(0) ; W(1)=1+\tilde{V}(0) ;$ and $W(w+y)=w+y-1+\tilde{V}(1)$ for all $w+y \geq 2$, we can show that (A1) is equivalent to (8) in the main text. There are no firstorder conditions, other than (8), that yield additional solutions. In particular, consider the following first-order condition: $\pi(2 ; 1,0)+\pi(3 ; 1,0)=1$ and (A5) with the strict equality if $\bar{\pi} \equiv \pi(3 ; 1,0)>0$. We can show that (A5) is equivalent to $\bar{\pi}=0$. Thus, only the corner solution is possible, but this only completes the corner solution in (8).

Derivation of (9), (10), and (11) is as discussed in the main text. From (2), (3), (9), and (10), we have:

$$
\tilde{V}(1)-\tilde{V}(0)=\beta(1-\delta)(V(1)-V(0))=\frac{\beta(1-\delta)(\tilde{\pi}(1-\tilde{\lambda})+\tilde{\lambda} / 2)}{1-\beta(1-\delta)(\tilde{\pi} \tilde{\lambda}+(1-\tilde{\lambda}) / 2)}
$$

If $\delta=0, \tilde{\lambda}=1$ in (11). Then, (A6) becomes:

$$
\tilde{V}(1)-\tilde{V}(0)=\frac{1}{2} \cdot \frac{\beta}{1-\beta \tilde{\pi}}
$$


In order to have $\tilde{V}(1)-\tilde{V}(0)>1$, in (A7) the probability $\tilde{\pi}$ needs to be sufficiently high:

$$
\tilde{\pi}>\frac{1}{\beta}-\frac{1}{2}
$$

If $\delta>0,(11)$ and (A6) become (12) and (13), respectively. Derivation of (14) is as discussed in the main text. Note that (14) nests (A8) as a special case of $\delta=0$.

In (8), $\tilde{\pi}$ is determined given $\tilde{V}(1)-\tilde{V}(0)$. If $\delta=0, \tilde{V}(1)-\tilde{V}(0)$ is determined given $\tilde{\pi}$ in $(\mathrm{A} 7)$. If $\delta>0, \tilde{\lambda}$ is determined given $\tilde{\pi}$ in $(12)$, and $\tilde{V}(1)-\tilde{V}(0)$ is determined given $\tilde{\lambda}$ in (13). Thus (8), (A7), (12), and (13) implicitly define a function, call it $\Gamma$, that takes a value of $\tilde{V}(1)-\tilde{V}(0)$ and returns another value of $\tilde{V}(1)-\tilde{V}(0)$. The equilibrium value of $\tilde{V}(1)-\tilde{V}(0)$ is the one that satisfies: $\Gamma(\tilde{V}(1)-\tilde{V}(0))=\tilde{V}(1)-\tilde{V}(0)$.

The derivation of (15) is as discussed in the main text. Assume that (15) holds from now on. Consulting (8) and (14), let $\Delta$ be the value of $\tilde{V}(1)-\tilde{V}(0)$ that solves:

$$
\pi_{0}=\frac{1}{2} \cdot\left(1-\Delta+\frac{1+\bar{u}}{\Delta}\right)
$$

Then, $\tilde{V}(1)-\tilde{V}(0)<\Delta$ in equilibrium if an equilibrium exists. A necessary condition for an equilibrium with $\tilde{V}(1)-\tilde{V}(0)>1$ is $\Delta>1$, which is equivalent to (16). Assume that (16) holds from now on. Let $\Lambda$ be the value of $\tilde{V}(1)-\tilde{V}(0)$ that yields $\tilde{\pi}=1$ in (8):

$$
1=\frac{1}{2} \cdot\left(1-\Lambda+\frac{1+\bar{u}}{\Lambda}\right)
$$

In (8), (A7), (12), and (13), observe that $\Gamma(\tilde{V}(1)-\tilde{V}(0))=\Gamma(\Lambda)$ for all $\tilde{V}(1)-\tilde{V}(0) \leq \Lambda$. If $\Lambda<\tilde{V}(1)-\tilde{V}(0)<\Delta, \tilde{\pi}$ decreases as $\tilde{V}(1)-\tilde{V}(0)$ increases in (8). If $\delta=0, \tilde{V}(1)-\tilde{V}(0)$ decreases as $\tilde{\pi}$ decreases in (A7). If $\delta>0, \tilde{\lambda}$ increases as $\tilde{\pi}$ decreases in (12), and $\tilde{V}(1)-\tilde{V}(0)$ decreases as $\tilde{\lambda}$ increases in (13). Thus, $\Gamma$ is decreasing in $\tilde{V}(1)-\tilde{V}(0)$ if $\Lambda<\tilde{V}(1)-\tilde{V}(0)<$ $\Delta$. Consulting (14) and (16), we have $\Gamma(1)>1$ and $\Gamma(\Delta)=1$. Further, $\Gamma$ is continuous 
and non-increasing if $1<\tilde{V}(1)-\tilde{V}(0)<\Delta$. Therefore, there is a unique fixed point in $(1, \Delta)$. Noting that $\Delta<1+\bar{u}$ in (A9), we have Proposition 3 .

\section{Proof of Proposition 4}

Consider first-order conditions when $\left(w, w^{\prime}\right)=(1,0)$ in (4). A first-order condition is $\pi(1 ; 1,0)+\pi(2 ; 1,0)=1$ and $(\mathrm{A} 1)$ with the strict equality if $\tilde{\pi} \equiv \pi(2 ; 1,0)<1$. Using that $W(0)=-\bar{u}+\tilde{V}(0) ; W(1)=-\bar{u}+\tilde{V}(1) ;$ and $W(w+y)=w+y-1+\tilde{V}(1)$, we can show that (A1) is equivalent to

$$
\tilde{\pi} \leq \frac{1}{2} \cdot\left(\frac{\tilde{V}(1)+\tilde{V}(0)}{1+\bar{u}}-\bar{u}\right)
$$

There are no first-order conditions, other than (A10), that yield additional solutions. In particular, consider the following first-order condition: $\pi(2 ; 1,0)+\pi(3 ; 1,0)=1$ and (A5) with the strict equality if $\bar{\pi} \equiv \pi(3 ; 1,0)>0$. We can show that (A5) is equivalent to $\bar{\pi}=0$. Thus, only the corner solution is possible, but this only completes the corner solution in (A10).

We have:

$$
\begin{aligned}
& V(0)=\left(\frac{1-\tilde{\lambda}}{2}+\tilde{\lambda} \cdot \tilde{\pi}\right)(-\bar{u}+\tilde{V}(1))+\left(\frac{1-\tilde{\lambda}}{2}+\tilde{\lambda} \cdot(1-\tilde{\pi})\right)(1+\tilde{V}(1)) ; \\
& V(1)=\left((1-\tilde{\lambda}) \cdot(1-\tilde{\pi})+\frac{\tilde{\lambda}}{2}\right)(1+\tilde{V}(1))+\left((1-\tilde{\lambda}) \cdot \tilde{\pi}+\frac{\tilde{\lambda}}{2}\right)(2+\tilde{V}(1)) .
\end{aligned}
$$

Since everyone saves one apple in equilibrium, $\tilde{\lambda} \equiv \lambda(1)=1-\delta$. Then, we have:

$$
\tilde{V}(1)-\tilde{V}(0)=\beta(1-\delta)(V(1)-V(0))=\beta(1-\delta)\left(\tilde{\pi}(1+\bar{u}(1-\delta))+\frac{1+\delta \bar{u}}{2}\right)
$$

Substituting (A11) in (A10), we have:

$$
\tilde{\pi} \leq \frac{1}{2} \cdot \frac{\beta(1-\delta)(1+\delta \bar{u})-2 \bar{u}(1+\bar{u})}{2(1+\bar{u})-\beta(1-\delta)(1+\bar{u}(1-\delta))}<\frac{1}{2}
$$


Substituting (A12) in (A11), we have:

$$
\tilde{V}(1)-\tilde{V}(0)<\beta(1-\delta)\left(1+\frac{\bar{u}}{2}\right)<1+\bar{u}
$$

This is a contradiction. Therefore, we have Proposition 4.

\section{Proof of Proposition 5}

It was shown in the main text that $\Omega>0$ when $\delta=0$. If $\delta>0$, given (17), we can express $\tilde{V}_{s}(1)$ in terms of $\beta, \delta$, and $\tilde{\lambda}$ only, using (12) and (13):

$$
\tilde{V}_{s}(1)=\frac{\beta}{1-\beta} \cdot\left(1+\left(\frac{1}{2 \tilde{\lambda}}-\frac{\delta}{1-\delta}\right)\left(1-\frac{1}{\beta /(1-\tilde{\lambda})+(1-\beta) / \delta)}\right)\right) .
$$

Given $\beta$ and $\delta$, the remaining parameter $\bar{u}$ determines $\tilde{\lambda}$ in the range $\left[\lambda_{1}, \lambda_{0}\right]$, corresponding to a $\tilde{\pi}$ in the range $\left[\tilde{\pi}_{0}, 1\right]$ according to (12). A higher $\bar{u}$ leads to a higher $\tilde{\pi}$ and a lower $\tilde{\lambda}$ within the ranges. Taking the partial derivative of $\tilde{V}_{s}(1)$ with respect to $\tilde{\lambda}$, we can show:

$$
\frac{\partial \tilde{V}_{s}(1)}{\partial \tilde{\lambda}}<\frac{\beta}{1-\beta} \cdot \frac{1}{2 \tilde{\lambda}^{2}}\left(\frac{1}{\beta+(1-\beta)(1-\tilde{\lambda}) / \delta}-1\right)<0
$$

at any $\tilde{\lambda} \in\left[\lambda_{1}, \lambda_{0}\right]$. The second inequality uses the relation $1-\tilde{\lambda}>\delta$ that can be derived from (12), (14), and (15) as follows. Define $\bar{\lambda}$ to be the solution to (12) assuming $\tilde{\pi}=1 / 2$ : $\bar{\lambda}=(1-\delta) /(1+\delta)$. Since $\tilde{\pi}>1 / 2$ in $(14), \tilde{\lambda}<\bar{\lambda}$ in (12). Then, $1-\tilde{\lambda}>1-\bar{\lambda}=2 \delta /(1+\delta)>\delta$ since $\delta<1 / 3$ in (15). Thus, holding $\beta$ and $\delta, \tilde{V}_{s}(1)$ decreases in $\tilde{\lambda}$ or, equivalently, increases in $\bar{u}$. The maximum value of $\tilde{V}_{s}(1)$ is when $\tilde{\lambda}=\lambda_{1}, \tilde{\pi}=1$, and $\bar{u} \geq \bar{u}_{1}$. Then, $\Omega \geq \Omega_{1}$, where $\Omega_{1}$ denote the value of $\Omega$ when $\bar{u} \geq \bar{u}_{1}$ given $\beta$ and $\delta$. From (A13), we have:

$$
\Omega_{1}=1-\frac{\beta}{1-\beta} \cdot\left(\left(\frac{1}{2 \lambda_{1}}-\frac{\delta}{1-\delta}\right)\left(1-\frac{1}{\left.\beta /\left(1-\lambda_{1}\right)+(1-\beta) / \delta\right)}\right)-\frac{1}{2}\right) .
$$

Setting $\tilde{\pi}=1$ in (12), we can derive:

$$
\delta=\frac{\left(1-\lambda_{1}\right)^{2}}{\left(1+\lambda_{1}^{2}\right)}
$$


Note that $\lambda_{1}$ depends only on $\delta$ and not on $\beta$ or $\bar{u}$. Now, we can substitute the above expression of $\delta$ in terms of $\lambda_{1}$ in the expression of $\Omega_{1}$ in (A14), and take the partial derivative of $\Omega_{1}$ with respect to $\beta$. Doing so, we can show that, for any $\delta$ with $\lambda_{1}$ given by (A15), $\Omega_{1}$ takes the minimum value when

$$
\beta=\max \left\{\frac{2}{3(1-\delta)}, \frac{1}{1+\delta}-\left(\frac{1}{(1+\delta)^{2}}-\frac{1-\delta-\delta /\left(1-\lambda_{1}\right)}{(1+\delta)\left(1-\delta /\left(1-\lambda_{1}\right)\right)}\right)^{1 / 2}\right\} .
$$

The first argument is the minimum given by (15) and the second argument is always less than 1. Finally, we can show that $\Omega_{1}$ increases in $\delta$ in (A14) when $\lambda_{1}$ and $\beta$ are given by (A15) and (A16), and $\Omega_{1} \rightarrow 0$ as $\delta \rightarrow 0$. Therefore, $\Omega_{1}>0$ for any $\beta$ and $\delta$. Then, we have: $\Omega \geq \Omega_{1}>0$ for any $\beta, \delta$, and $\bar{u}$. Therefore, a would-be-rich person becomes better off upon the implementation of the no-saving policy.

It is shown in the main text that a would-be-poor person also becomes better off, as summarized in (19). Therefore, we have Proposition 5.

\section{Proof of Proposition 6}

Equations (20) and (21) are consistent with the following consumption/saving pattern: $c=w+y$ and $s=0$ if $w+y \leq 1$; and $c=w+y-1$ and $s=1$ if $w+y \geq 2$. As a proof, observe that $U(w+y)-U(w+y-s)=s(1+\bar{u}) \geq s \cdot \beta(V(1)-V(0)) \geq \beta(V(s)-V(0))$ if $s \leq w+y \leq 1$; and $U(w+y-s)-U(w+y-1)=1-s \leq(1-s) \cdot \beta(V(1)-V(0)) \leq \beta(V(1)-V(s))$ if $w+y \geq 2$ and $s \leq 1$. This consumption/saving pattern is equivalent to:

$$
W(w+y)=-\bar{u}+(1+\bar{u}) \cdot(w+y)+\beta V(0)
$$

if $w+y \leq 1$; and

$$
W(w+y)=w+y-1+\beta V(1)
$$

if $w+y \geq 2$. 
Further, (20) implies: $U(c)-U(1)=c-1 \leq(c-1) \cdot \beta(V(1)-V(0))$ if $c \geq 1$; and $U(c)-U(1)=-(1-c)(1+\bar{u}) \leq(c-1) \cdot \beta(V(1)-V(0))$ if $c \leq 1$. For all $w+y \in[1,2]$, using (21), we have: $W(w+y))-W(1)=\max _{c}\{U(c)-U(1)+\beta(V((w+y-c)-V(0)\} \leq$ $\max _{c}\{(c-1) \cdot \beta(V(1)-V(0))+(w+y-c) \cdot \beta(V(1)-V(0))\}=(w+y-1) \cdot \beta(V(1)-V(0))=$ $(w+y-1) \cdot(W(2)-W(1))$ or equivalently,

$$
W(w+y) \leq(w+y-1) \cdot W(2)+(2-w-y) \cdot W(1)
$$

for all $w+y \in[1,2]$. Then, the bargained sum of wealth and income, $w+y$, may probabilistically take on the value of 1 or 2 .

Further, (A17) and (A18) imply:

$$
W(w+y)-W(w)=y(1+\bar{u}) \geq y \cdot \beta(V(1)-V(0))=y(W(2)-W(1))
$$

for all $w \in[0,1]$ and $y \in[0,1-w]$; and

$W(w+y)-W(2)=w+y-2 \leq(w+y-2) \cdot \beta(V(1)-V(0))=(w+y-2)(W(2)-W(1))$

for all $w \in[0,1]$ and $y \in[2-w, 3]$. Then, possible output shares can be represented as a convex set with linear frontiers in the first quadrant of a two dimensional space with the axes of $\sum_{y} \pi(y ; w, 1)$ and $\sum_{y} \pi(y ; 1, w)$.

Redrawing the frontiers of possible output shares as $w$ changes, we can conjecture the following pattern of the bargaining outcome: there are $\tilde{w}_{1}, \tilde{w}_{2}$, and $\tilde{w}_{3}$ with $0<\tilde{w}_{1}<$ $\tilde{w}_{2}<\tilde{w}_{3}<1$ such that $\pi\left(\tilde{y}_{1}(w) ; w, 1\right)=1$ if $0 \leq w \leq \tilde{w}_{1} ; \pi\left(\tilde{y}_{1}(w) ; w, 1\right)+\pi\left(\tilde{y}_{2}(w) ; w, 1\right)=1$ if $\tilde{w}_{1} \leq w \leq \tilde{w}_{2} ; \pi\left(\tilde{y}_{2}(w) ; w, 1\right)=1$ if $\tilde{w}_{2} \leq w \leq \tilde{w}_{3} ; \pi\left(\tilde{y}_{3}(w) ; w, 1\right)=1$ if $\tilde{w}_{3} \leq w \leq 1$, where $\tilde{y}_{1}(w), \tilde{y}_{2}(w)$, and $\tilde{y}_{3}(w)$ solve: $w+\tilde{y}_{1}(w)=1 ; w+\tilde{y}_{2}(w)=2$;

$$
w+\tilde{y}_{3}(w)=2+\frac{w-\tilde{w}_{3}}{2\left(1-\tilde{w}_{3}\right)}
$$


and

$$
\pi\left(\tilde{y}_{2}(w) ; w, 1\right)=\frac{w-\tilde{w}_{1}}{\tilde{w}_{2}-\tilde{w}_{1}}
$$

Given the assumed consumption/saving behavior, we have: $V(1)=3 / 2+\beta V(1)=3 /(2(1-$ $\beta)) ; V(w)=w+\tilde{y}_{3}(w)-1+\beta V(1)$ if $\tilde{w}_{3} \leq w \leq 1 ; V(w)=1+\beta V(1)=(2+\beta) /(2(1-\beta))$ if $\tilde{w}_{2} \leq w \leq \tilde{w}_{3}$

$$
V(w)=1+\beta\left(\pi\left(\tilde{y}_{2}(w) ; w, 1\right) \cdot V(1)+\left(1-\pi\left(\tilde{y}_{2}(w) ; w, 1\right)\right) \cdot V(0)\right)
$$

if $\tilde{w}_{1} \leq w \leq \tilde{w}_{2} ;$ and $V(w)=1+\beta V(0)=1 /(1-\beta)$ if $0 \leq w \leq \tilde{w}_{1}$.

From the above expressions of $V(w)$, observe that $\beta(V(1)-V(0))=\beta /(2(1-\beta))$. Then, (20) is equivalent to:

$$
1 \leq \frac{\beta}{2(1-\beta)} \leq 1+\bar{u}
$$

From the above expressions of $V(w),(21)$ is equivalent to:

$$
\beta\left(V\left(\tilde{w}_{2}\right)-V(0)\right) \leq \tilde{w}_{2} \cdot \beta(V(1)-V(0))
$$

or to:

$$
\tilde{w}_{2} \geq \frac{\beta\left(V\left(\tilde{w}_{2}\right)-V(0)\right)}{\beta(V(1)-V(0))}=\beta .
$$

Now, I will derive the expressions of $\tilde{w}_{1}, \tilde{w}_{2}$, and $\tilde{w}_{3}$. If $\tilde{w}_{3} \leq w \leq 1$, the first order condition of maximizing the Nash product is:

$$
W\left(4-\tilde{y}_{3}(w)\right)-W(1)-\left(W\left(w+\tilde{y}_{3}(w)\right)-W(w)\right)=0 .
$$

Using (A18), we have:

$$
\tilde{y}_{3}(w)=\frac{3}{2}-\frac{\bar{u}(1-w)}{2} .
$$

Comparing (A19) and (A22), we have:

$$
\tilde{w}_{3}=\frac{1+\bar{u}}{2+\bar{u}}
$$


If $\tilde{w}_{1} \leq w \leq \tilde{w}_{2}$, the first order condition of maximizing the Nash product is:

$$
\begin{aligned}
& (W(2)-W(1)) \cdot(\pi(2-w ; w, 1) \cdot W(2+w)+(1-\pi(2-w ; w, 1)) \cdot W(3+w)-W(1)) \\
+ & (W(2+w)-W(3+w)) \cdot(\pi(2-w ; w, 1) \cdot W(2)+(1-\pi(2-w ; w, 1)) \cdot W(1)-W(w))=0 .
\end{aligned}
$$

Since $\pi\left(2-\tilde{w}_{2} ; \tilde{w}_{2}, 1\right)=1$, we have:

$$
\beta(V(1)-V(0)) \cdot\left(\tilde{w}_{2}+\beta(V(1)-V(0))\right)=(1+\bar{u})\left(1-\tilde{w}_{2}\right)+\beta(V(1)-V(0))
$$

or

$$
\tilde{w}_{2}=1-\frac{\beta^{2}(V(1)-V(0))^{2}}{1+\bar{u}+\beta(V(1)-V(0))} .
$$

Similarly, since $\pi\left(2-\tilde{w}_{1} ; \tilde{w}_{1}, 1\right)=0$, we have:

$$
\beta(V(1)-V(0)) \cdot\left(1+\tilde{w}_{1}+\beta(V(1)-V(0))\right)=(1+\bar{u})\left(1-\tilde{w}_{1}\right)
$$

or

$$
\tilde{w}_{1}=\frac{1+\bar{u}-\beta(V(1)-V(0))-\beta^{2}(V(1)-V(0))^{2}}{1+\bar{u}+\beta(V(1)-V(0))} .
$$

Given (A23), (A21) becomes (22). We can verify that $0<\tilde{w}_{1}<\tilde{w}_{2}<\tilde{w}_{3}<1$ given (A20) and (22). Finally, observe that the second inequality in (A20) holds if (22) holds and the first inequality is equivalent to $(15)^{\prime}$. In summary, we have Proposition 6.

\section{Proof of Proposition 7}

Equations (23), (24), and (25) are consistent with the following saving/consumption pattern: $W(0)=-\bar{u}+\beta V(0) ; W(w+y)=1+\beta V(w+y-1)$ if $w+y \leq \bar{w}+1$; and $W(w+y)=w+y-\bar{w}+\beta V(\bar{w})$ if $w+y \geq \bar{w}+1$.

For $\left(w, w^{\prime}\right)=(\bar{w}, \bar{w}+n)$ with $n \geq 1$, I can show that, holding $\pi(0 ; \bar{w}, \bar{w}+n)+\pi(1 ; \bar{w}, \bar{w}+n)=$ 1 , the first order derivative of the Nash Product in $(4)^{\prime \prime}$ with respect to $\pi(1 ; \bar{w}, \bar{w}+n)$ is 
positive for all values of $\pi(1 ; \bar{w}, \bar{w}+n)$. Therefore, $\pi(0 ; \bar{w}, \bar{w}+n)=0$. Then, the first order condition is $\pi(1 ; \bar{w}, \bar{w}+n)+\pi(2 ; \bar{w}, \bar{w}+n)=1$ and

$$
\begin{aligned}
& (W(\bar{w}+2)-W(\bar{w}+1))(\check{\pi}(\bar{w}+n) W(\bar{w}+n+1)+(1-\check{\pi}(\bar{w}+n)) W(\bar{w}+n+2)-W(\bar{w}+n)) \\
+ & (W(\bar{w}+n+1)-W(\bar{w}+n+2))(\check{\pi}(\bar{w}+n) W(\bar{w}+2)+(1-\check{\pi}(\bar{w}+n)) W(\bar{w}+1)-W(\bar{w})) \leq 0
\end{aligned}
$$

with the strict equality if $\check{\pi}(\bar{w}+n) \equiv \pi(2 ; \bar{w}, \bar{w}+n)>0$.

For $\left(w, w^{\prime}\right)=(\bar{w}, \bar{w}-n)$ with $n \geq 1$, I can show that, holding $\pi(2 ; \bar{w}, \bar{w}-n)+\pi(3 ; \bar{w}, \bar{w}-n)=$ 1, the first order derivative of the Nash Product in $(4)^{\prime \prime}$ with respect to $\pi(3 ; \bar{w}, \bar{w}-n)$ is negative for all values of $\pi(3 ; \bar{w}, \bar{w}-n)$. Therefore, $\pi(3 ; \bar{w}, \bar{w}-n)=0$. Then, the first order condition is $\pi(1 ; \bar{w}, \bar{w}-n)+\pi(2 ; \bar{w}, \bar{w}-n)=1$ and

$$
\begin{aligned}
& (W(\bar{w}+2)-W(\bar{w}+1))(\check{\pi}(\bar{w}-n) W(\bar{w}-n+1)+(1-\check{\pi}(\bar{w}-n)) W(\bar{w}-n+2)-W(\bar{w}-n)) \\
+ & (W(\bar{w}-n+1)-W(\bar{w}-n+2))(\check{\pi}(\bar{w}-n) W(\bar{w}+2)+(1-\check{\pi}(\bar{w}-n)) W(\bar{w}+1)-W(\bar{w})) \geq 0
\end{aligned}
$$

with the strict equality if $\check{\pi}(\bar{w}-n) \equiv \pi(2 ; \bar{w}, \bar{w}-n)<1$.

Given the expressions of $W(w),(\mathrm{A} 24)$ is equivalent to:

$$
\check{\pi}(\bar{w}+n) \geq 1-\frac{\beta(V(\bar{w})-V(\bar{w}-1))}{2}
$$

for $n \geq 1$. Observe that the right-hand side of (A26) is free of $n$. Then, $\check{\pi}(\bar{w}+n)=\check{\pi}(\bar{w}+m)$ and $\hat{\pi}(\bar{w}+n)=\hat{\pi}(\bar{w}+m)$ for all $n, m \geq 1$. Then, $\beta(V(\bar{w}+n)-V(\bar{w}+n-1))=\beta<1$ for all $n \geq 2$. This verifies (25) for $n \geq 2$.

Given the expressions of $W(w),(\mathrm{A} 25)$ is equivalent to:

$$
\check{\pi}(0) \leq \frac{1}{2}\left(1-\beta(V(\bar{w})-V(\bar{w}-1))+\frac{1+\bar{u}}{\beta(V(1)-V(0))}\right)
$$

and

$$
\check{\pi}(\bar{w}-n) \leq \frac{1}{2}\left(1-\beta(V(\bar{w})-V(\bar{w}-1))+\frac{\beta(V(\bar{w}-n)-V(\bar{w}-n-1))}{\beta(V(\bar{w}-n+1)-V(\bar{w}-n))}\right)
$$


for $n \in\{1,2, \ldots, \bar{w}-1\}$.

Now, the value functions can be written as:

$$
\begin{gathered}
V(\bar{w}-n)=1+\beta(\check{\pi}(\bar{w}-n) \cdot V(\bar{w}-n)+(1-\check{\pi}(\bar{w}-n)) \cdot V(\bar{w}-n+1)) ; \\
V(\bar{w})=\frac{3}{2}+\beta V(\bar{w}) ;
\end{gathered}
$$

and

$$
V(\bar{w}+n)=\check{\pi}(\bar{w}+1) \cdot(n+1)+(1-\check{\pi}(\bar{w}+1)) \cdot(n+2)+\beta V(\bar{w}),
$$

where $n \geq 1$.

In order for the first inequality in (23) to hold for $n=0$, in (A28) and (A29), we need:

$$
\beta(V(\bar{w})-V(\bar{w}-1))=\frac{\beta}{2(1-\beta \check{\pi}(\bar{w}-1))} \geq 1
$$

or

$$
\check{\pi}(\bar{w}-1) \geq \pi_{0} \equiv \frac{1}{\beta}-\frac{1}{2} .
$$

Since $\check{\pi}(\bar{w}-1) \leq 1$, a necessary condition for the equilibrium with $\bar{w} \geq 1$ is:

$$
\beta \geq \frac{2}{3}
$$

In order for (25) to hold for $n=1$, in (A29) and (A30), we need:

$$
\beta(V(\bar{w}+1)-V(\bar{w}))=\beta\left(\frac{3}{2}-\check{\pi}(\bar{w}+1)\right) \leq 1
$$

or

$$
\check{\pi}(\bar{w}+1) \geq \frac{3}{2}-\frac{1}{\beta} .
$$


From (A26) and (A31), $\check{\pi}(\bar{w}+1) \geq 1-\beta /(4(1-\beta))$. Combined with (A33), this implies that $\check{\pi}(\bar{w}+1)>0$ given (15). Then, (A26) holds with equality. Then, from (A33), (A31), and (A26), we have:

$$
\check{\pi}(\bar{w}-1) \leq \pi_{1} \equiv \min \left\{1, \frac{1}{\beta}-\frac{1}{2} \cdot \frac{\beta}{2-\beta}\right\} .
$$

The equilibrium value of $\check{\pi}(\bar{w}-1)$ is limited to the range $\left[\pi_{0}, \pi_{1}\right]$, given (14) and (A34).

In order for (24) to hold, in (A28) and (A29), further we need:

$$
\frac{\beta(V(\bar{w}-n)-V(\bar{w}-n-1))}{\beta(V(\bar{w}-n+1)-V(\bar{w}-n))}=\frac{\beta(1-\check{\pi}(\bar{w}-n))}{1-\beta \check{\pi}(\bar{w}-n-1)} \geq 1
$$

or

$$
\check{\pi}(\bar{w}-n-1)-\check{\pi}(\bar{w}-n) \geq \frac{1}{\beta}-1
$$

for all $n \in\{1,2, \ldots, \bar{w}-1\}$.

Note that (A36) implies that $\check{\pi}(\bar{w}-n)<1$ for all $n \in\{1,2, \ldots, \bar{w}-1\}$. Then, (A27) holds with equality. Then, from (A31), (A35), and (A27), we have:

$$
\frac{\beta(1-\check{\pi}(\bar{w}-n))}{1-\beta \check{\pi}(\bar{w}-n-1)}=2 \check{\pi}(\bar{w}-n)-1+\frac{\beta}{2(1-\beta \check{\pi}(\bar{w}-1))},
$$

which yields $\check{\pi}(\bar{w}-n-1)$ given $\check{\pi}(\bar{w}-n)$.

Assume (15) and consider the following algorithm of finding the equilibrium values. Pick a value of $\check{\pi}(\bar{w}-1)$ between $\pi_{0}$ and $\pi_{1}$ in (14) and (A34). Then, (A32) and (A31) hold by construction. Derive $\{\check{\pi}(\bar{w}-n)\}$ for $n \geq 2$ from (26). From (A31) and (14), observe that the righthand side of (26) is greater than 1. Then, (A36) holds. Given (A36), observe that there is $\bar{n} \geq 1$ so that $\check{\pi}(\bar{w}-\bar{n}) \leq 1$ and there is no $\check{\pi}(\bar{w}-\bar{n}-1) \leq 1$ that solves (26). Then, the maximum possible equilibrium value of $\bar{w}$ is $\bar{n}$. For any $\bar{w} \leq \bar{n}$, derive $\bar{u}$ that solves (27) with equality given (A31) and (A35). Given (14), (15), and (A36), $\check{\pi}(0) \geq \pi_{0}>1 / 2$. Given (A31), $\beta(V(\bar{w})-V(\bar{w}-1)>1$. Then, $1+\bar{u}>\beta(V(1)-V(0))$ in $(27)$. This verifies 
the second inequality in (23). Finally, set $\check{\pi}(\bar{w})=1 / 2$; derive $\check{\pi}(\bar{w}+1)$ from (A26) and (A31); and set $\check{\pi}(\bar{w}+n)=\check{\pi}(\bar{w}+1)$ for all $n \geq 2$. Then, by construction, $\bar{w}, \bar{u}$, and $\{\check{\pi}(n)\}_{n \geq 0}$ are an equilibrium.

The above algorithm shows that any $\bar{w} \in\{1,2, \ldots, \bar{n}\}$, where $\bar{n}$ is determined by $\check{\pi}(\bar{w}-1)$, is supported as an equilibrium by some value of $\bar{u}$. In order to keep track of the various equilibria, let $\bar{n} \mid \check{\pi}(\bar{w}-1)$ denote $\bar{n}$ given $\check{\pi}(\bar{w}-1)$. Let $\check{\pi}(w) \mid(\bar{w}, \check{\pi}(\bar{w}-1))$ denote $\check{\pi}(w)$ given $\bar{w}$ and $\check{\pi}(\bar{w}-1)$. Similarly, let $\bar{u} \mid(\bar{w}, \check{\pi}(\bar{w}-1))$ denote $\bar{u}$ that solves $(27)$ with equality, given $\bar{w}$ and $\check{\pi}(\bar{w}-1)$. Let $V(w) \mid(\bar{w}, \check{\pi}(\bar{w}-1))$ denote $V(w)$ given $\bar{w}$ and $\check{\pi}(\bar{w}-1)$.

Observe that $\check{\pi}(w+1)|(\bar{w}+1, \check{\pi}(\bar{w}-1))=\check{\pi}(w)|(\bar{w}, \check{\pi}(\bar{w}-1))>\check{\pi}(w+1) \mid(\bar{w}, \check{\pi}(\bar{w}-1))$ if $w, w+1 \in\{1, \bar{n} \mid \check{\pi}(\bar{w}-1)\}$. In particular, $\check{\pi}(0)|(\bar{w}+1, \check{\pi}(\bar{w}-1))>\check{\pi}(0)|(\bar{w}, \check{\pi}(\bar{w}-1))$. Similarly, we can show that $V(1)|(\bar{w}+1, \check{\pi}(\bar{w}-1))-V(0)|(\bar{w}+1, \check{\pi}(\bar{w}-1))>V(1) \mid(\bar{w}, \check{\pi}(\bar{w}-$ 1)) $-V(0) \mid(\bar{w}, \check{\pi}(\bar{w}-1))$. Then, $\bar{u}|(\bar{w}+1, \check{\pi}(\bar{w}-1))>\bar{u}|(\bar{w}, \check{\pi}(\bar{w}-1))$ in $(27)$ if $w, w+1 \in$ $\{1, \bar{n} \mid \check{\pi}(\bar{w}-1)\}$.

Observe that $\check{\pi}(\bar{w}-n-1)$ as a function of $\check{\pi}(\bar{w}-n)$ is continuous and increasing in (26) so that $\check{\pi}(w) \mid(\bar{w}, \check{\pi}(\bar{w}-1))$ is continuous and increasing in $\check{\pi}(\bar{w}-1)$, holding $w$ and $\bar{w}$, while $\check{\pi}(\bar{w}-1) \in\left[\pi_{0}, \pi_{1}\right]$. Relatedly, the lefthand side of (26) or, equivalently, the righthand side of $(\mathrm{A} 35)$ as a function of $\check{\pi}(\bar{w}-1)$ is continuous and increasing. Consulting (A31), observe that $V(1)|(\bar{w}, \check{\pi}(\bar{w}-1))-V(0)|(\bar{w}, \check{\pi}(\bar{w}-1))$ is continuous and increasing in $\check{\pi}(\bar{w}-1)$. Putting these properties together, observe that $\bar{u} \mid(\bar{w}, \check{\pi}(\bar{w}-1))$ is continuous and increasing in $\check{\pi}(\bar{w}-1)$ in (27). Then, if $\bar{w} \leq \bar{n} \mid \pi_{1}$ and $\bar{u}\left|\left(\bar{w}, \pi_{0}\right)<\bar{u}<\bar{u}\right|\left(\bar{w}, \pi_{1}\right)$ for some $\bar{u}$, there is $x \in\left(\pi_{0}, \pi_{1}\right)$ so that $\bar{w} \leq \bar{n} \mid x$ and $\bar{u} \mid x=\bar{u}$. Therefore, for any $\bar{w} \leq \bar{n} \mid \pi_{1}$ and $\bar{u} \in\left[\bar{u}\left|\left(\bar{w}, \pi_{0}\right), \bar{u}\right|\left(\bar{w}, \pi_{1}\right)\right]$, there is $\check{\pi}(\bar{w}-1)$ that is an equilibrium along with $\{\check{\pi}(w)\}$ derived by the above algorithm.

Now suppose that $\check{\pi}(0) \mid\left(\bar{n} \mid \pi_{1}, \pi_{1}\right)=1$. Then, in (27), observe that any $\bar{u} \geq \bar{u} \mid\left(\bar{n} \mid \pi_{1}, \pi_{1}\right)$ along with the other equilibrium values associated with $\check{\pi}(\bar{w}-1)=\pi_{1}$ is an equilibrium 
by construction. Therefore, the range of $\bar{u}$ that supports the equilibrium with $\bar{w}=\bar{n} \mid \pi_{1}$ is extended to the infinite set $\left[\bar{u} \mid\left(\bar{n}, \pi_{0}\right), \infty\right)$ if $\check{\pi}(0) \mid\left(\bar{n} \mid \pi_{1}, \pi_{1}\right)=1$. Note that, for $\bar{w}<$ $\bar{n} \mid \pi_{1}$, the range of $\bar{u}$ that supports the equilibrium with $\bar{w}$ is necessarily the finite set $\left[\bar{u}\left|\left(\bar{w}, \pi_{0}\right), \bar{u}\right|\left(\bar{w}, \pi_{1}\right)\right]$ since $\bar{u} \mid\left(\bar{w}, \pi_{1}\right)$ is increasing in $\bar{w}$.

Now suppose that $\bar{n}\left|\pi_{1}<\bar{n}\right| \pi_{0}$. Then, for any $\bar{w} \in\left\{\bar{n}\left|\pi_{1}+1, \bar{n}\right| \pi_{0}\right\}, \check{\pi}(0) \mid\left(\bar{w}, \pi_{0}\right)$ and $\bar{u} \mid\left(\bar{w}, \pi_{0}\right)$ exist while $\check{\pi}(0) \mid\left(\bar{w}, \pi_{1}\right)$ and $\bar{u} \mid\left(\bar{w}, \pi_{1}\right)$ do not exist. Since $\bar{u} \mid(\bar{w}, \check{\pi}(\bar{w}-1))$ is continuous and increasing in $\check{\pi}(\bar{w}-1)$, there is $\check{\pi}(\bar{w}-1) \in\left[\pi_{0}, \pi_{1}\right]$, call it $\pi_{2}(\bar{w})$, so that $\check{\pi}(0) \mid\left(\bar{w}, \pi_{2}(\bar{w})\right)=1$. Then, any $\bar{u} \in\left[\bar{u}\left|\left(\bar{w}, \pi_{0}\right), \bar{u}\right|\left(\bar{w}, \pi_{2}(\bar{w})\right)\right]$ supports some $\check{\pi}(\bar{w}-1) \in$ $\left[\pi_{0}, \pi_{2}(\bar{w})\right]$ as an equilibrium by the above algorithm. Further, any $\bar{u} \geq \bar{u} \mid\left(\bar{w}, \pi_{2}(\bar{w})\right)$ supports $\check{\pi}(\bar{w}-1)=\pi_{2}(\bar{w})$ as an equilibrium, repeating the reasoning in the above. Therefore, for any $\bar{w} \in\left\{\bar{n}\left|\pi_{1}+1, \bar{n}\right| \pi_{0}\right\}$, the range of $\bar{u}$ that supports the equilibrium with $\bar{w}$ is necessarily the infinite set $\left[\bar{u} \mid\left(\bar{w}, \pi_{0}\right), \infty\right)$.

In the remainder of the proof, we will show that the union of the ranges of $\bar{u}$ that support the equilibria with various values of $\bar{w}$ is contiguous, i.e., if $x_{1}<x_{2}$ and if there are an equilibrium with $\bar{u}=x_{1}$ and another equilibrium with $\bar{u}=x_{2}$, there is an equilibrium with $\bar{u}=x$ for any $x \in\left(x_{1}, x_{2}\right)$. The ranges of $\bar{u}$ that support the equilibria with $\bar{w} \in$ $\left\{\bar{n}\left|\pi_{1}+1, \bar{n}\right| \pi_{0}\right\}$, if any, are the infinite sets, so the union of these sets is contiguous. If $\check{\pi}(0) \mid\left(\bar{n} \mid \pi_{1}, \pi_{1}\right)=1$, the range of $\bar{u}$ that supports the equilibrium with $\bar{w}=\bar{n} \mid \pi_{1}$ is also infinite so that the union of the ranges of $\bar{u}$ that support the equilibria with $\bar{w} \in\left\{\bar{n}\left|\pi_{1}, \bar{n}\right| \pi_{0}\right\}$ is contiguous. Therefore, the union of the ranges of $\bar{u}$ that support any equilibria is contiguous if $\bar{u}\left|\left(\bar{w}, \pi_{0}\right)<\bar{u}\right|\left(\bar{w}-1, \pi_{1}\right)$ for any $\bar{w}$ with $\bar{w} \leq \bar{n}\left|\pi_{0}, \bar{w}-1 \leq \bar{n}\right| \pi_{1}$, and $\check{\pi}(0) \mid\left(\bar{w}-1, \pi_{1}\right)<1$.

If $\pi_{1}=1, \bar{n} \mid \pi_{1}=1$ and $\check{\pi}(0) \mid\left(\bar{n} \mid \pi_{1}, \pi_{1}\right)=\pi_{1}=1$ so that the union of the ranges of $\bar{u}$ that support any equilibria is contiguous. Consider the case of $\pi_{1}<1$ in the following. From 
(A35) and (26), we have:

$$
\frac{\beta(V(\bar{w}-n)-V(\bar{w}-n-1))}{\beta(V(\bar{w}-n+1)-V(\bar{w}-n))}=2 \check{\pi}(\bar{w}-n)-1+\frac{\beta}{2(1-\beta \check{\pi}(\bar{w}-1))} .
$$

Equation (A37) holds for $n \geq 1$; it also holds for $n=0$ if $\check{\pi}(\bar{w}-1)=\pi_{1}$ since $\check{\pi}(\bar{w})=1 / 2$ and $\beta(V(\bar{w}+1)-V(\bar{w}))=1$ if $\check{\pi}(\bar{w}-1)=\pi_{1}$, given (A32), (A33), and (A34). From (A31) and (14), $\beta /\left(2\left(1-\beta \pi_{0}\right)\right)=1$. From (A26), (A33), (A31), (14), and (A34), $\beta /\left(2\left(1-\beta \pi_{1}\right)\right)=2 \pi_{0}$. Then, from (A37), we have:

$$
\frac{\Delta(w-1) \mid\left(\bar{w}-1, \pi_{1}\right)}{\Delta(w-1) \mid\left(\bar{w}, \pi_{0}\right)}=\frac{2 \check{\pi}(w) \mid\left(\bar{w}-1, \pi_{1}\right)+2 \pi_{0}-1}{2 \check{\pi}(w) \mid\left(\bar{w}, \pi_{0}\right)} \cdot \frac{\Delta(w) \mid\left(\bar{w}-1, \pi_{1}\right)}{\Delta(w) \mid\left(\bar{w}, \pi_{0}\right)},
$$

where $w \leq \bar{w}-1, \Delta(w) \mid(\bar{w}, \check{\pi}(\bar{w}-1)) \equiv \beta(V(w+1)|(\bar{w}, \check{\pi}(\bar{w}-1))-V(w)|(\bar{w}, \check{\pi}(\bar{w}-1)))$, and $V(w) \mid(\bar{w}, \check{\pi}(\bar{w}-1))$ is $V(w)$ given $\bar{w}$ and $\check{\pi}(\bar{w}-1)$. From $(26)$, we can derive:

$$
2 \check{\pi}(w-1) \mid\left(\bar{w}, \pi_{0}\right)=\frac{2}{\beta}+1-\frac{2}{2 \check{\pi}(w) \mid\left(\bar{w}, \pi_{0}\right)}
$$

and

$$
2 \check{\pi}(w-1) \mid\left(\bar{w}, \pi_{1}\right)+2 \pi_{0}-1=\frac{2}{\beta}+2 \pi_{0}-\frac{2 \pi_{0}+1}{2 \check{\pi}(w) \mid\left(\bar{w}, \pi_{1}\right)+2 \pi_{0}-1} .
$$

Note that (A39) and (A40) define two functions, $F$ and $G: 2 \check{\pi}(w-1) \mid\left(\bar{w}, \pi_{0}\right)=F(2 \check{\pi}(w) \mid(\bar{w}$, $\left.\left.\pi_{0}\right)\right)$ and $2 \check{\pi}(w-1) \mid\left(\bar{w}, \pi_{1}\right)+2 \pi_{0}-1=G\left(2 \check{\pi}(w) \mid\left(\bar{w}, \pi_{1}\right)+2 \pi_{0}-1\right)$. Further, note that $F(z)<G(z)$ for all $z \geq 1$. Let $F^{t+1}(z) \equiv F\left(F^{t}(z)\right)$ and $G^{t+1}(z) \equiv G\left(G^{t}(z)\right)$ for all $t \geq 1$. Then, from (A38), we have:

$$
\begin{aligned}
\frac{\Delta(w) \mid\left(\bar{w}-1, \pi_{1}\right)}{\Delta(w) \mid\left(\bar{w}, \pi_{0}\right)} & =\frac{G^{\bar{w}-w-1}\left(2 \check{\pi}(\bar{w}-1) \mid\left(\bar{w}-1, \pi_{1}\right)+2 \pi_{0}-1\right)}{F^{\bar{w}-w-1}\left(2 \check{\pi}(\bar{w}-1) \mid\left(\bar{w}, \pi_{0}\right)\right)} \cdot \frac{\Delta(\bar{w}-1) \mid\left(\bar{w}-1, \pi_{1}\right)}{\Delta(\bar{w}-1) \mid\left(\bar{w}, \pi_{0}\right)} \\
& =\frac{G^{\bar{w}-w-1}\left(2 \pi_{0}\right)}{F^{\bar{w}-w-1}\left(2 \pi_{0}\right)} \\
& >1,
\end{aligned}
$$

where the second equality uses: $\check{\pi}(\bar{w}-1)\left|\left(\bar{w}-1, \pi_{1}\right)=1 / 2 ; \check{\pi}(\bar{w}-1)\right|\left(\bar{w}, \pi_{0}\right)=\pi_{0}$; $\Delta(\bar{w}-1) \mid\left(\bar{w}-1, \pi_{1}\right)=\beta\left(V(\bar{w})\left|\left(\bar{w}-1, \pi_{1}\right)-V(\bar{w}-1)\right|\left(\bar{w}-1, \pi_{1}\right)\right)=1 ;$ and $\Delta(\bar{w}-1) \mid\left(\bar{w}, \pi_{0}\right)=$ $\beta\left(V(\bar{w})\left|\left(\bar{w}, \pi_{0}\right)-V(\bar{w}-1)\right|\left(\bar{w}, \pi_{0}\right)\right)=1$. From (27), (A38), and (A41), we have:

$$
\frac{1+\bar{u} \mid\left(\bar{w}-1, \pi_{1}\right)}{1+\bar{u} \mid\left(\bar{w}, \pi_{0}\right)}=\frac{2 \check{\pi}(0) \mid\left(\bar{w}-1, \pi_{1}\right)+2 \pi_{0}-1}{2 \check{\pi}(0) \mid\left(\bar{w}, \pi_{0}\right)} \cdot \frac{\Delta\left(0 \mid\left(\bar{w}-1, \pi_{1}\right)\right)}{\Delta\left(0 \mid\left(\bar{w}, \pi_{0}\right)\right)}=\frac{G^{\bar{w}}\left(2 \pi_{0}\right)}{F^{\bar{w}}\left(2 \pi_{0}\right)}>1
$$


if $\bar{w} \leq \bar{n} \mid \pi_{0}$ and $\bar{w}-1 \leq \bar{n} \mid \pi_{1}$. Therefore, the union of the ranges of $\bar{u}$ that support any equilibria is contiguous.

In order to summarize, let $\bar{\omega} \equiv \bar{n}\left|\pi_{0} ; \check{u}(\bar{w}) \equiv \bar{u}\right|\left(\bar{w}, \pi_{0}\right)$; and $\hat{u}(\bar{w}) \equiv \bar{u} \mid\left(\bar{w}, \pi_{1}\right)$. We then have Proposition 7. 


\section{Working Paper Series}

ISSN 1211-3298

Registration No. (Ministry of Culture): E 19443

Individual researchers, as well as the on-line and printed versions of the CERGE-EI Working Papers (including their dissemination) were supported from institutional support RVO 67985998 from Economics Institute of the ASCR, v. v. i.

Specific research support and/or other grants the researchers/publications benefited from are acknowledged at the beginning of the Paper.

(c) Byeongju Jeong, 2013

All rights reserved. No part of this publication may be reproduced, stored in a retrieval system or transmitted in any form or by any means, electronic, mechanical or photocopying, recording, or otherwise without the prior permission of the publisher.

Published by

Charles University in Prague, Center for Economic Research and Graduate Education (CERGE) and

Economics Institute ASCR, v. v. i. (EI)

CERGE-El, Politických vězňů 7, 11121 Prague 1, tel.: +420 224005 153, Czech Republic.

Printed by CERGE-EI, Prague

Subscription: CERGE-EI homepage: http://www.cerge-ei.cz

Phone: + 420224005153

Email: office@cerge-ei.cz

Web: http://www.cerge-ei.cz

Editor: Michal Kejak

The paper is available online at http://www.cerge-ei.cz/publications/working_papers/.

ISBN 978-80-7343-283-6 (Univerzita Karlova. Centrum pro ekonomický výzkum a doktorské studium)

ISBN 978-80-7344-275-0 (Národohospodářský ústav AV ČR, v. v. i.) 
CERGE-EI

P.O.BOX 882

Politických vězňů 7

11121 Praha 1

Czech Republic http://www.cerge-ei.cz 